MATHEMATICS OF COMPUTATION

Volume 77, Number 264, October 2008, Pages 1917-1939

S 0025-5718(08)02116-9

Article electronically published on April 28, 2008

\title{
ROBUST A POSTERIORI ERROR ESTIMATION FOR THE NONCONFORMING FORTIN-SOULIE FINITE ELEMENT APPROXIMATION
}

\author{
MARK AINSWORTH AND RICHARD RANKIN
}

\begin{abstract}
We obtain a computable a posteriori error bound on the broken energy norm of the error in the Fortin-Soulie finite element approximation of a linear second order elliptic problem with variable permeability. This bound is shown to be efficient in the sense that it also provides a lower bound for the broken energy norm of the error up to a constant and higher order data oscillation terms. The estimator is completely free of unknown constants and provides a guaranteed numerical bound on the error.
\end{abstract}

\section{INTRODUCTION}

A posteriori error estimation plays a key role in the assessment of the accuracy of finite element simulations and in the control of adaptive refinement algorithms. Although the theory of a posteriori error estimation for conforming finite element methods is now relatively well understood [3, 5, 22, the development of corresponding techniques for nonconforming finite element approximation is less so.

Various alternative a posteriori estimators for the error in the broken energy norm have been proposed for the lowest order nonconforming Crouzeix-Raviart finite element approximation on triangular elements, including explicit residualbased estimators [11, hierarchical basis estimators [14 and averaging type estimators [10]. All of these estimators provide two-sided bounds on the error up to unknown positive constants that are nevertheless independent of the mesh-size. A hybrid averaging/residual based estimator proposed in [1] was shown to provide actual computable numerical bounds on the error in the broken energy norm and, again up to a generic positive constant independent of the mesh-size, local lower bounds on the error. Moreover, the dependence of the estimator on jumps in the diffusion coefficient was studied and shown to be relatively mild.

The second order, nonconforming finite element of Fortin and Soulie [13] offers some advantages over the first order Crouzeix-Raviart element, perhaps most important is that it satisfies a discrete Korn inequality 8. Explicit residual-type a posteriori error estimators for the Fortin-Soulie element were developed in [7] in

Received by the editor October 10, 2006 and, in revised form, April 5, 2007.

2000 Mathematics Subject Classification. Primary 65N15, 65N30.

Key words and phrases. Robust a posteriori error estimation, nonconforming finite element, Fortin-Soulie element.

Partial support of the first author by the Engineering and Physical Sciences Research Council of Great Britain under grant GR/S35101 and of the second author through a research studentship is gratefully acknowledged. 
the context of linear elasticity, and in [19] in the setting of quasi-Newtonian flow. However, as yet there are no available a posteriori error estimators that provide actual numerical bounds on the error along with local lower bounds.

Our objective in the present work is to address this issue and, moreover, to study the effectiveness of the estimator when the diffusion coefficient is allowed to undergo large jumps from element to element. In particular, we wish to investigate whether the approach adopted in [1] for the Crouzeix-Raviart element may be generalised to the Fortin-Soulie element. At first glance, this appears unlikely since the approach used in [1 implicitly takes advantage of a number of rather special properties of the Crouzeix-Raviart element 16 that are not inherited by the Fortin-Soulie element. In particular, advantage is taken in [1] of the fact that local fluxes may be defined that are in equilibrium with the interior residual over each individual elementthe so-called equilibrated fluxes [3]. While a similar property holds for the FortinSoulie element, following the approach of [1] would lead to an estimator that indeed delivers a computable upper bound, but one which becomes increasingly pessimistic were the mesh to be refined. Nevertheless, by carrying out suitable modifications, we are able to construct a readily computable upper bound on the error that also provides local lower bounds up to a generic constant. Consequently, the estimator provides fully reliable, quantitative error control along with efficiency. In addition, the dependence of the estimator on jumps in the diffusion coefficient is obtained under weaker conditions than those imposed in 6, 21] in the context of conforming finite element approximation where monotonicity requirements are imposed on the diffusion coefficients. Here, as in [1, no such requirements are imposed.

The remainder of the paper is organised as follows. In Section 2, we describe the finite element scheme and introduce the notation. Following [1, 11] we decompose the broken energy norm of the error into conforming and nonconforming components. Upper and lower bounds for each of these components are derived in Sections 3 and 4 . Finally, in Section 5 we summarise the main results and present some numerical examples illustrating the theory.

\section{Preliminaries}

2.1. Model Problem. Consider the model problem

$$
-\operatorname{div}(a \operatorname{grad} u)=f \text { in } \Omega
$$

subject to $u=q$ on $\Gamma_{D}$ and $a \frac{\partial u}{\partial \hat{n}}=g$ on $\Gamma_{N}$, where $\Omega$ is a simple plane polygonal domain, and the disjoint sets $\Gamma_{D}$ (nonempty) and $\Gamma_{N}$ form a partitioning of the boundary $\Gamma=\partial \Omega$ of the domain. The data satisfy $f \in L_{2}(\Omega), g \in L_{2}\left(\Gamma_{N}\right)$, $q \in H^{1}\left(\Gamma_{D}\right)$, and $a \in L_{\infty}(\Omega)$ is piecewise constant. We will assume that $a$ is positive and that the value of $a$ across the boundary of a subdomain of $\Omega$ may undergo a jump of many orders of magnitude, corresponding to transition between regions of widely differing permeability.

The variational form of the problem consists of finding $u \in H^{1}(\Omega)$ such that $u=q$ on $\Gamma_{D}$ and

$$
(a \operatorname{grad} u, \operatorname{grad} v)=(f, v)+(g, v)_{\Gamma_{N}} \forall v \in H_{E}^{1}(\Omega),
$$

where $H_{E}^{1}(\Omega)=\left\{v \in H^{1}(\Omega): v=0\right.$ on $\left.\Gamma_{D}\right\}$. We shall use the notation $(\cdot, \cdot)_{\omega}$ to denote the integral inner product over a region or line segment $\omega$, and omit the subscript in the case where $\omega$ is the physical domain $\Omega$. 
Consider a family of partitions $\{\mathcal{P}\}$ of the domain $\Omega$ into the union of nonoverlapping, shape regular triangular elements such that the nonempty intersection of a distinct pair of elements is a single common node or single common edge. Consequently, the family of partitions is locally quasi-uniform in the sense that the ratio of the diameters of any pair of neighbouring elements is uniformly bounded above and below on the whole family.

2.2. Notation. In order to facilitate the orientation, we summarise all of the notations used throughout the manuscript with reference to the place where they are first used.

Section 2.1: $\Omega, \Gamma_{D}, \Gamma_{N}, \Gamma, f, g, q, a, u, H_{E}^{1},(\cdot, \cdot)_{\omega},(\cdot, \cdot), \mathcal{P}$.

Section 2.3: $K, K^{\prime}, \partial \mathcal{P}, \gamma, \gamma^{\prime}, \gamma^{\prime \prime}, \partial K, \mathbb{P}_{n}(K), \mathbb{P}_{n}(\gamma), \xi_{K},[v]_{\gamma}, X^{n c}, X_{E}^{n c}, u_{n c}$, $\operatorname{grad}_{\mathbf{n c}}, q_{I}$.

Section 2.4: $|K|,|\gamma|, h_{K}, \mathcal{V}, \lambda_{n}, \boldsymbol{x}_{m}, \mathcal{V}(K), \mathcal{V}(\gamma), \bar{v}_{K}, \bar{v}_{\gamma}, \hat{\boldsymbol{t}}_{\gamma}^{K}, \hat{\boldsymbol{n}}_{\gamma}^{K}, s_{\gamma}^{K}, \theta_{K}, \theta_{\gamma}$, $\Pi, C_{K}, C_{\gamma}^{K}$.

Section 2.5: osc $(f, K), P_{K} f, \operatorname{osc}(g, \gamma), P_{\gamma} g, H_{\gamma}^{1}(K), \operatorname{osc}(q, \gamma)$.

Section 2.6: $a_{K}, \mathcal{N}, \mathcal{N}(K), \mathcal{N}\left(\Gamma_{D}\right), \Omega_{n}, \wp\left(K, K^{\prime}\right), \wp^{\star}\left(K, K^{\prime}\right), a_{K K^{\prime}}, \wp\left(K, \Gamma_{D}, n\right)$, $\wp^{\star}\left(K, \Gamma_{D}, n\right), a_{K \Gamma_{D}, n}, \Upsilon_{K K^{\prime}}, \Upsilon_{K \Gamma_{D}}, \Upsilon_{K}, \tilde{K}$

Section 2.7: $\|\cdot\|_{\omega},\|\cdot\|, e, \mathcal{H}, \phi, \psi$.

Section 3.1: $\left[a \frac{\partial u_{n c}}{\partial \hat{\boldsymbol{n}}}\right]_{\gamma}, \boldsymbol{x}_{K}, \alpha_{\gamma}^{K}, \boldsymbol{t}_{i}, \boldsymbol{n}_{i}, \boldsymbol{\theta}_{1}^{K}, \boldsymbol{\theta}_{2}^{K}, \boldsymbol{\theta}_{3}^{K}, \boldsymbol{\vartheta}^{K}, \boldsymbol{\sigma}_{K}, \beta_{K}, \Phi_{K}\left(\beta_{K}\right), \boldsymbol{\sigma}_{K}^{\star}$.

Section 3.2: $\vec{x}_{K}, \vec{y}_{K}, \boldsymbol{M}, \boldsymbol{v}^{K}, \boldsymbol{M}_{i}, \beta_{\gamma}^{K}, \hat{\boldsymbol{t}}_{\gamma}, v_{\gamma}, \hat{K}$.

Section 4.1: $\mathcal{S}\left(u_{n c}\right), \omega_{K, n}, \Psi_{K}$.

Section 4.2: $\partial \Omega_{n}, \mu, \beta_{\gamma}, \partial \wp^{*}\left(K, K^{\prime}\right), \beta_{K K^{\prime}}, \beta_{K \Gamma_{D}, n}, \mathcal{E}_{K}$.

Section 5.1: $\vec{V}, \boldsymbol{S}, Q, \boldsymbol{A}, \boldsymbol{B}, \boldsymbol{C}$.

Section 5.2: Ndofs, $\eta_{n}, \beta_{K}^{(n)}, \vartheta_{n}$.

2.3. Nonconforming finite element approximation. Let $K$ and $K^{\prime}$ denote individual elements in $\mathcal{P}$, let $\partial \mathcal{P}$ denote the set of element edges, let $\gamma, \gamma^{\prime}$ and $\gamma^{\prime \prime}$ denote individual edges in $\partial \mathcal{P}$ and let $\partial K$ denote the set of edges of element $K$. Let $\mathbb{P}_{n}(K)$ denote the space of polynomials on $K \in \mathcal{P}$ of total degree at most $n$ and let $\mathbb{P}_{n}(\gamma)$ denote the space of polynomials on $\gamma \subset \partial \mathcal{P}$ of total degree at most $n$. For each element $K \in \mathcal{P}$, let $\xi_{K}: \partial K \rightarrow\{+1,-1\}$ denote a sign function that is piecewise constant on $\partial K$ and chosen such that $\xi_{K}+\xi_{K^{\prime}}=0$ on $\partial K \cap \partial K^{\prime}$. For $v \in X_{E}^{n c}$, we define the jump of $v$ on an edge $\gamma$ by $[v]_{\gamma}=\xi_{K} v_{\mid K}+\xi_{K^{\prime}} v_{\mid K^{\prime}}$ if $\gamma=\partial K \cap \partial K^{\prime}$. The Fortin-Soulie finite element space is defined by

$$
\begin{aligned}
X^{n c}= & \left\{v: \Omega \rightarrow \mathbb{R}: v_{\mid K} \in \mathbb{P}_{2}(K) \forall K \in \mathcal{P},\right. \\
& \left.\left([v]_{\gamma}, p\right)_{\gamma}=0 \forall p \in \mathbb{P}_{1}(\gamma) \text { for } \gamma \subset \partial \mathcal{P} \backslash \Gamma\right\}
\end{aligned}
$$

with the subspace $X_{E}^{n c}$ defined by

$$
X_{E}^{n c}=\left\{v \in X^{n c}:(v, p)_{\gamma}=0 \forall p \in \mathbb{P}_{1}(\gamma) \text { for } \gamma \subset \Gamma_{D}\right\} .
$$

Note that although these definitions seem to differ from the usual way of defining the Fortin-Soulie finite element spaces the above are exactly the same spaces as those defined in [13, 15] since for all $\gamma \subset \partial \mathcal{P} \backslash \Gamma$, if $v \in X_{E}^{n c}$, then $[v]_{\gamma} \in \mathbb{P}_{2}(\gamma)$ is orthogonal to $\mathbb{P}_{1}(\gamma)$, and hence $v$ is continuous at the two Gauss-Legendre points 


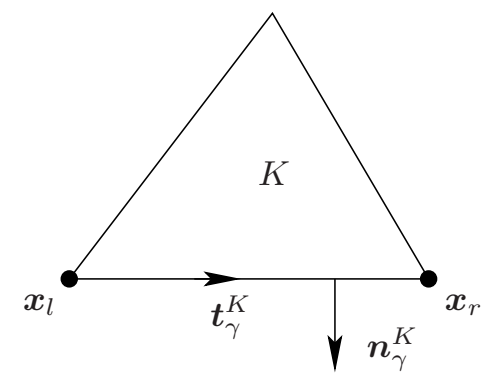

FiguRe 1. Location of the endpoints and orientation of the tangent and normal vectors on an edge $\gamma$ of element $K$.

on $\gamma$. Also, for all $\gamma \subset \partial \mathcal{P} \cap \Gamma_{D}$, if $v \in X_{E}^{n c}$, then $v_{\mid \gamma} \in \mathbb{P}_{2}(\gamma)$ is orthogonal to $\mathbb{P}_{1}(\gamma)$, and hence $v$ is zero at the two Gauss-Legendre points on $\gamma$.

The nonconforming finite element approximation of problem (2.1) consists of finding $u_{n c} \in X^{n c}$ such that

$$
\begin{aligned}
\left(a \operatorname{grad}_{\mathbf{n c}} u_{n c}, \operatorname{grad}_{\mathbf{n c}} v\right) & =(f, v)+(g, v)_{\Gamma_{N}} \forall v \in X_{E}^{n c}, \\
\left(u_{n c}, p\right)_{\gamma} & =\left(q_{I}, p\right)_{\gamma} \forall p \in \mathbb{P}_{1}(\gamma) \text { for } \gamma \subset \Gamma_{D}
\end{aligned}
$$

where $\operatorname{grad}_{\mathbf{n c}}$ denotes the operator defined by $\left(\operatorname{grad}_{\mathbf{n c}} v\right)_{\mid K}=\operatorname{grad}\left(v_{\mid K}\right)$ for $K \in$ $\mathcal{P}$. Here, the Dirichlet datum $q$ has been approximated by the piecewise quadratic interpolant $q_{I}$ which equals $q$ at the endpoints and midpoint of each edge $\gamma \in \partial \mathcal{P}$ on the Dirichlet boundary $\Gamma_{D}$.

2.4. Projection operator. Let $|K|$ denote the area of the element $K$, let $|\gamma|$ denote the length of edge $\gamma$ and let $h_{K}$ be the length of the longest edge of element $K$. Let $\mathcal{V}$ index the set of element vertices and for $n \in \mathcal{V}$ define $\lambda_{n}$ to be the piecewise linear function which satisfies $\lambda_{n}\left(\boldsymbol{x}_{m}\right)=\delta_{m n} \forall m \in \mathcal{V}$ where $\boldsymbol{x}_{m}$ denotes the position vector of vertex $m$. For $K \in \mathcal{P}$ let $\mathcal{V}(K)$ index the set of the vertices of element $K$ and for $\gamma \subset \partial \mathcal{P}$ let $\mathcal{V}(\gamma)$ index the vertices at the endpoints of $\gamma$. Throughout we shall make use of the following formulae:

$$
\left(\lambda_{i}^{l} \lambda_{j}^{m} \lambda_{k}^{n}, 1\right)_{K}=\frac{2 l ! m ! n !}{(l+m+n+2) !}|K|
$$

for $l, m, n \geq 0$ where $\mathcal{V}(K)=\{i, j, k\}$ and, with $\mathcal{V}(\gamma)=\{l, r\}$, for $m, n \geq 0$,

$$
\left(\lambda_{l}^{m}, \lambda_{r}^{n}\right)_{\gamma}=\frac{m ! n !}{(m+n+1) !}|\gamma| .
$$

Let $\bar{v}_{K}=\frac{1}{|K|}(v, 1)_{K}$ for $v \in L_{2}(K)$, let $\bar{v}_{\gamma}=\frac{1}{|\gamma|}(v, 1)_{\gamma}$ for $v \in L_{2}(\gamma)$ and let the tangent vector $\boldsymbol{t}_{\gamma}^{K}$ and normal vector $\boldsymbol{n}_{\gamma}^{K}$ to edge $\gamma$ of element $K$ be oriented as shown in Figure 1, with the corresponding unit vectors denoted by $\hat{\boldsymbol{t}}_{\gamma}^{K}$ and $\hat{\boldsymbol{n}}_{\gamma}^{K}$.

We may parametrise an edge $\gamma \subset \partial K$ by $\boldsymbol{x}\left(s_{\gamma}^{K}\right)=\boldsymbol{x}_{l}+\left(s_{\gamma}^{K}+\frac{|\gamma|}{2}\right) \hat{\boldsymbol{t}}_{\gamma}^{K}$ where

$$
s_{\gamma}^{K}=\frac{|\gamma|}{2}\left(\lambda_{r}-\lambda_{l}\right) \in\left(-\frac{|\gamma|}{2}, \frac{|\gamma|}{2}\right) \text {. }
$$


For future reference we note that $s_{\gamma}^{K}$ has the properties that for $p \in \mathbb{P}_{1}(\gamma)$,

$$
p-\bar{p}_{\gamma}=\frac{\partial p}{\partial \hat{\boldsymbol{t}}_{\gamma}^{K}} s_{\gamma}^{K} \text { and } \frac{\partial s_{\gamma}^{K}}{\partial \hat{\boldsymbol{t}}_{\gamma}^{K}}=1 .
$$

Lemma 2.1. Let $\theta_{K} \in X_{E}^{n c}$ be defined by

$$
\theta_{K}= \begin{cases}4-6 \sum_{n \in \mathcal{V}(K)} \lambda_{n}^{2} & \text { on } K, \\ 0, & \text { elsewhere },\end{cases}
$$

and for $\gamma \subset \partial K$ let $\theta_{\gamma} \in X^{n c}$ be defined by

$$
\theta_{\gamma}= \begin{cases}1-6\left(1-\sum_{n \in \mathcal{V}(\gamma)} \lambda_{n}\right) \sum_{n \in \mathcal{V}(\gamma)} \lambda_{n} & \text { on } K, \\ 0, & \text { elsewhere. }\end{cases}
$$

Define the operator $\Pi: H_{E}^{1}(\Omega) \rightarrow X_{E}^{n c}$ by

$$
\Pi v=\sum_{K \in \mathcal{P}} \bar{v}_{K} \theta_{K}+\sum_{\gamma \subset \partial \mathcal{P}} \bar{v}_{\gamma} \theta_{\gamma}
$$

Then for all $K \in \mathcal{P}$,

$$
(v-\Pi v, 1)_{K}=0,
$$

and for all $\gamma \subset \partial \mathcal{P}$,

$$
(v-\Pi v, p)_{\gamma}=\left(v, p-\bar{p}_{\gamma}\right)_{\gamma} \forall p \in \mathbb{P}_{1}(\gamma) .
$$

Also,

$$
\|v-\Pi v\|_{L_{2}(K)} \leq C_{K}\|\operatorname{grad} v\|_{L_{2}(K)}
$$

where for $\gamma, \gamma^{\prime}$ distinct edges

$$
C_{K}=\frac{h_{K}}{\pi}+\sum_{\gamma \subset \partial K}\left(\frac{1}{5} \frac{h_{K}}{\pi}\left(\frac{h_{K}}{\pi}+\max _{\gamma^{\prime} \subset \partial K}\left|\gamma^{\prime}\right|\right)\right)^{1 / 2},
$$

and for $\gamma \subset \partial K \cap \Gamma_{N}$,

$$
\|v-\Pi v\|_{L_{2}(\gamma)} \leq C_{\gamma}^{K}\|\operatorname{grad} v\|_{L_{2}(K)}
$$

where for $\gamma^{\prime}, \gamma^{\prime \prime}$ distinct edges

$$
C_{\gamma}^{K}=\frac{|\gamma|^{1 / 2}}{|K|^{1 / 2}} \sum_{\gamma^{\prime} \subset \partial K}\left(\left(2 \delta_{\gamma \gamma^{\prime}}+\frac{1}{5} \frac{|\gamma|}{\left|\gamma^{\prime}\right|}\left(1-\delta_{\gamma \gamma^{\prime}}\right)\right) \frac{h_{K}}{\pi}\left(\frac{h_{K}}{\pi}+\max _{\gamma^{\prime \prime} \subset \partial K}\left|\gamma^{\prime \prime}\right|\right)\right)^{1 / 2} .
$$

Proof. By applying (2.4), we obtain $\left(\theta_{K}, 1\right)_{K}=|K|$ and $\left(\theta_{\gamma}, 1\right)_{K}=0$, from which (2.11) follows. Likewise, applying (2.5) we obtain $\left(\theta_{K}, p\right)_{\gamma^{\prime}}=0$ and $\left(\theta_{\gamma}, p\right)_{\gamma^{\prime}}=$ $|\gamma| \bar{p}_{\gamma} \delta_{\gamma \gamma^{\prime}} \forall p \in \mathbb{P}_{1}\left(\gamma^{\prime}\right)$ for all $\gamma, \gamma^{\prime} \subset \partial \mathcal{P}$ from which (2.12) follows. Moreover, these identities show that $\Pi c=c$ on $K$ for all $c \in \mathbb{R}$ and since for $v \in H^{1}(K)$, there exists $c \in \mathbb{R}$ such that [20], $\|v-c\|_{L_{2}(K)} \leq \frac{h_{K}}{\pi}\|\operatorname{grad} v\|_{L_{2}(K)}$ and [2],

$$
\|v-c\|_{L_{2}(\gamma)} \leq\left(\frac{|\gamma|}{|K|} \frac{h_{K}}{\pi}\left(\frac{h_{K}}{\pi}+\max _{\gamma^{\prime} \subset \partial K}\left|\gamma^{\prime}\right|\right)\right)^{1 / 2}\|\operatorname{grad} v\|_{L_{2}(K)}
$$


for $\gamma, \gamma^{\prime}$ distinct edges, by applying standard arguments, 2.13) and (2.15) follow.

2.5. Data oscillation. The oscillation of the data $f$ on an element $K \in \mathcal{P}$ is defined to be

$$
\operatorname{osc}(f, K)=|K|^{1 / 2} \inf _{p \in \mathbb{P}_{1}(K)}\|f-p\|_{L_{2}(K)} .
$$

Since $\mathbb{P}_{1}(K)$ is finite-dimensional and the norm is strictly convex, it follows that there exists a unique minimiser of the right hand side, which we shall denote by $P_{K} f$. Likewise, the oscillation of the Neumann data $g$ on an edge $\gamma \subset \Gamma_{N}$ is defined to be

$$
\operatorname{osc}(g, \gamma)=|\gamma|^{1 / 2} \inf _{p \in \mathbb{P}_{1}(\gamma)}\|g-p\|_{L_{2}(\gamma)}
$$

and again we denote the minimiser by $P_{\gamma} g$. For $K \in \mathcal{P}$ and $\gamma \subset \partial K \cap \Gamma_{D}$, let the space $H_{\gamma}^{1}(K)=\left\{v: v \in H^{1}(K): v=0\right.$ on $\left.\partial K \backslash \gamma\right\}$. The oscillation of the Dirichlet data $q$ on an edge $\gamma \subset \partial K \cap \Gamma_{D}$ is defined to be

$$
\operatorname{osc}(q, \gamma)=\inf _{\substack{v \in H_{\gamma}^{1}(K): \\ v_{\mid \gamma}=q-q_{I}}}\|\operatorname{grad} v\|_{L_{2}(K)}
$$

2.6. Path permeability. Let $a_{K}$ denote the value taken by the permeability $a$ on element $K$, let $\mathcal{N}$ index the set of element vertices and edge midpoints, let $\mathcal{N}(K)$ index the set of vertices and edge midpoints of element $K$, let $\mathcal{N}\left(\Gamma_{D}\right)$ index the set of element vertices and edge midpoints which lie on $\overline{\Gamma_{D}}$, for $n \in \mathcal{N}$ let $\Omega_{n}$ denote the patch composed from those elements with a vertex or edge midpoint located at $\boldsymbol{x}_{n}$ and let $K, K^{\prime} \subset \Omega_{n}$ be distinct elements. To define the permeability $a_{K K^{\prime}}$ between a pair of elements, first observe that there is always at least one connected path $\wp\left(K, K^{\prime}\right) \subset \mathcal{P}$ passing from $K$ to $K^{\prime}$ through adjacent elements belonging to the patch $\Omega_{n}$. The smallest permeability of all the elements in the path $\wp\left(K, K^{\prime}\right)$ is given by $\min \left\{a_{M}: M \in \wp\left(K, K^{\prime}\right)\right\}$. We take $\wp^{\star}\left(K, K^{\prime}\right)$ to be the path which maximises the value of this quantity, and define

$$
a_{K K^{\prime}}=\min \left\{a_{M}: M \in \wp^{\star}\left(K, K^{\prime}\right)\right\} .
$$

If a vertex or edge midpoint $\boldsymbol{x}_{n}$ of element $K$ lies on $\overline{\Gamma_{D}}$, then the element may be linked to $\Gamma_{D}$ by a connected path $\wp\left(K, \Gamma_{D}, n\right)$ passing through adjacent elements as before. Choosing $\wp^{\star}\left(K, \Gamma_{D}, n\right)$ to be the path which maximises the value of $\min \left\{a_{M}: M \in \wp\left(K, \Gamma_{D}, n\right)\right\}$, the permeability between element $K$ and the Dirichlet boundary is then defined by $a_{K \Gamma_{D}, n}=\min \left\{a_{M}: M \in \wp^{\star}\left(K, \Gamma_{D}, n\right)\right\}$. The ratio $\Upsilon_{K K^{\prime}}=\frac{\min \left(a_{K}, a_{K^{\prime}}\right)}{a_{K K^{\prime}}}$ measures path permeability relative to the least permeable of the two elements $K$ and $K^{\prime}$ at the endpoints of the path and $\Upsilon_{K \Gamma_{D}}=$ $\sum_{n \in \mathcal{N}(K) \cap \mathcal{N}\left(\Gamma_{D}\right)} \frac{a_{K}}{a_{K \Gamma_{D}, n}}$. We also define

$$
\Upsilon_{K}=\Upsilon_{K \Gamma_{D}}+\sum_{K^{\prime} \subset \tilde{K} \backslash K} \Upsilon_{K K^{\prime}}
$$

where $\tilde{K}$ denotes the patch formed from element $K$ and those elements sharing a common vertex with element $K$. For more details and examples on path permeability we refer the reader to 1 . 
2.7. A posteriori error estimator. Let the broken energy norm over a region $\omega$ be denoted by $\|\cdot\|_{\omega}=\left(a \operatorname{grad}_{\mathbf{n c}} \cdot, \operatorname{grad}_{\mathbf{n c}} \cdot\right)_{\omega}^{1 / 2}$ where again we shall omit the subscript in the case where $\omega=\Omega$. Our objective is to obtain a computable estimator for the broken energy norm of the error $e=u-u_{n c}$. Observe that this also provides $L_{2}(\Omega)$ error control because of the discrete Poincaré-Friedrichs inequality. To estimate the error we shall make use of the following lemma, which is proved in [11] and used in [4, 9].

Lemma 2.2. Let $\mathcal{H}=\left\{w \in H^{1}(\Omega):(w, 1)_{\Omega}=0\right.$ and $\frac{\partial w}{\partial \hat{\boldsymbol{t}}}=0$ on $\left.\Gamma_{N}\right\}$. The error $e=u-u_{n c}$ may be decomposed into the form

$$
a \operatorname{grad}_{\mathbf{n c}} e=a \operatorname{grad} \phi+\operatorname{curl} \psi
$$

where the conforming error $\phi \in H_{E}^{1}(\Omega)$ satisfies

$$
(a \operatorname{grad} \phi, \operatorname{grad} v)=\left(a \operatorname{grad}_{\mathbf{n c}} e, \operatorname{grad} v\right) \forall v \in H_{E}^{1}(\Omega)
$$

and the nonconforming error $\psi \in \mathcal{H}$ satisfies

$$
\left(a^{-1} \operatorname{curl} \psi, \operatorname{curl} w\right)=\left(\operatorname{grad}_{\mathbf{n c}} e, \operatorname{curl} w\right) \forall w \in \mathcal{H} .
$$

Moreover,

$$
\|e\|^{2}=\|\phi\|^{2}+\left(a^{-1} \operatorname{curl} \psi, \operatorname{curl} \psi\right) .
$$

The importance of this lemma is that it allows us to write $\|e\|^{2}$ as the sum of a conforming part $\|\phi\|^{2}$ and a nonconforming part $\left(a^{-1} \operatorname{curl} \psi, \operatorname{curl} \psi\right)$ which reduces the problem of obtaining an estimator for $\|e\|$ to that of obtaining separate estimators for each of the two terms in this decomposition which is what we discuss in the next two sections.

\section{ESTIMATION OF THE CONFORMING ERROR}

3.1. Upper bound. Our aim is to find a fully computable upper bound for $\|\phi\|^{2}$. Let $v \in H_{E}^{1}(\Omega)$. Then, by (2.20), the definition of $e$ and (2.1),

$$
\begin{aligned}
& (a \operatorname{grad} \phi, \operatorname{grad} v)=\left(a \operatorname{grad}_{\mathbf{n c}} e, \operatorname{grad} v\right) \\
& \quad=(a \operatorname{grad} u, \operatorname{grad} v)-\left(a \operatorname{grad}_{\mathbf{n c}} u_{n c}, \operatorname{grad} v\right) \\
& \quad=(f, v)+(g, v)_{\Gamma_{N}}-\left(a \operatorname{grad}_{\mathbf{n c}} u_{n c}, \operatorname{grad} v\right) \\
& \quad=\sum_{K \in \mathcal{P}}\left((f, v)_{K}-\left(a a_{K} \operatorname{grad} u_{n c}, \operatorname{grad} v\right)_{K}+\sum_{\gamma \subset \partial K}(g, v)_{\gamma \cap \Gamma_{N}}\right) .
\end{aligned}
$$

Observing that $\Pi v \in X_{E}^{n c}$, where $\Pi$ is the operator defined in Lemma 2.1, we find

$$
0=\left(a \operatorname{grad}_{\mathbf{n c}} u_{n c}, \operatorname{grad}_{\mathbf{n c}} \Pi v\right)-(f, \Pi v)-(g, \Pi v)_{\Gamma_{N}}
$$

from (2.3), and so

$$
\begin{aligned}
(a \operatorname{grad} \phi, \operatorname{grad} v)= & \sum_{K \in \mathcal{P}}\left(-\left(a_{K} \operatorname{grad} u_{n c}, \operatorname{grad}(v-\Pi v)\right)_{K}\right. \\
& \left.+(f, v-\Pi v)_{K}+\sum_{\gamma \subset \partial K}(g, v-\Pi v)_{\gamma \cap \Gamma_{N}}\right) .
\end{aligned}
$$


Integrating by parts, applying property (2.12), and the fact that $v=0$ on $\Gamma_{D}$ gives

$$
\begin{aligned}
& (a \operatorname{grad} \phi, \operatorname{grad} v)=\sum_{K \in \mathcal{P}}\left(f+a_{K} \operatorname{div}\left(\operatorname{grad} u_{n c}\right), v-\Pi v\right)_{K} \\
& \left.+\sum_{\gamma \subset \partial \mathcal{P} \backslash \Gamma_{D}}\left(\left(g-P_{\gamma} g, v-\Pi v\right)_{\gamma \cap \Gamma_{N}}-\left(\left[a \frac{\partial u_{n c}}{\partial \hat{\boldsymbol{n}}}\right]_{\gamma}-\overline{\left(\left[a \frac{\partial u_{n c}}{\partial \hat{\boldsymbol{n}}}\right]_{\gamma}\right.}\right)_{\gamma}, v\right)\right)
\end{aligned}
$$

where

$$
\left[a \frac{\partial u_{n c}}{\partial \hat{\boldsymbol{n}}}\right]_{\gamma}= \begin{cases}a_{K} \frac{\partial u_{n c \mid K}}{\partial \hat{\boldsymbol{n}}_{\gamma}^{K}}+a_{K^{\prime}} \frac{\partial u_{n c \mid K^{\prime}}}{\partial \hat{\boldsymbol{n}}_{\gamma}^{K^{\prime}}} & \text { if } \gamma=\partial K \cap \partial K^{\prime}, \\ a_{K} \frac{\partial u_{n \mid K}}{\partial \hat{\boldsymbol{n}}_{\gamma}^{K}}-P_{\gamma} g & \text { if } \gamma \subset \Gamma_{N}, \\ a_{K} \frac{\partial u_{n c \mid K}}{\partial \hat{\boldsymbol{n}}_{\gamma}^{K}} & \text { if } \gamma \subset \Gamma_{D} .\end{cases}
$$

We now simplify the terms appearing in the right hand side as follows. First, since $a_{K} \operatorname{div}\left(\operatorname{grad}_{\mathbf{n c}} u_{n c}\right)$ is constant, property (2.11) of the operator $\Pi$ allows us to write

$$
\begin{aligned}
& \left(f+a_{K} \operatorname{div}\left(\operatorname{grad} u_{n c}\right), v-\Pi v\right)_{K} \\
& \quad=\left(\operatorname{grad}\left(P_{K} f\right) \cdot\left(\boldsymbol{x}-\boldsymbol{x}_{K}\right), v-\Pi v\right)_{K}+\left(f-P_{K} f, v-\Pi v\right)_{K}
\end{aligned}
$$

where $\boldsymbol{x}_{K}$ denotes the centroid of element $K \in \mathcal{P}$. The final term in (3.3) will be estimated later in terms of the data oscillation. For each $K \in \mathcal{P}$ and each edge $\gamma \subset \partial K$, define

$$
\alpha_{\gamma}^{K}= \begin{cases}\frac{a_{K^{\prime}}^{-1 / 2}}{a_{K}^{-1 / 2}+a_{K^{\prime}}^{-1 / 2}} & \text { if } \gamma=\partial K \cap \partial K^{\prime}, \\ 1 & \text { if } \gamma \subset \Gamma_{N}, \\ 0 & \text { if } \gamma \subset \Gamma_{D} .\end{cases}
$$

Then, since $\alpha_{\gamma}^{K}+\alpha_{\gamma}^{K^{\prime}}=1$ for $\gamma=\partial K \cap \partial K^{\prime}$,

$$
\begin{aligned}
\sum_{\gamma \subset \partial \mathcal{P} \backslash \Gamma_{D}}\left(\left[a \frac{\partial u_{n c}}{\partial \hat{\boldsymbol{n}}}\right]_{\gamma}-\overline{\left(\left[a \frac{\partial u_{n c}}{\partial \hat{\boldsymbol{n}}}\right]_{\gamma}\right)_{\gamma}}, v\right)_{\gamma} & =\sum_{K \in \mathcal{P}} \sum_{\gamma \subset \partial K}\left(\alpha_{\gamma}^{K}\left(\left[a \frac{\partial u_{n c}}{\partial \hat{\boldsymbol{n}}}\right]_{\gamma}-\left(\left[a \frac{\partial u_{n c}}{\partial \hat{\boldsymbol{n}}}\right]_{\gamma}\right)_{\gamma}\right), v\right)_{\gamma} \\
= & \sum_{K \in \mathcal{P}} \sum_{\gamma \subset \partial K}\left(\alpha_{\gamma}^{K} \frac{\partial}{\partial \hat{\boldsymbol{t}}_{\gamma}^{K}}\left[a \frac{\partial u_{n c}}{\partial \hat{\boldsymbol{n}}}\right]_{\gamma} s_{\gamma}^{K}, v\right)_{\gamma}
\end{aligned}
$$

upon applying property (2.7). Hence, with the aid of (3.3), we may rewrite (3.1) in the form

$$
\begin{aligned}
& (a \operatorname{grad} \phi, \operatorname{grad} v) \\
& =\sum_{K \in \mathcal{P}}\left(\left(\operatorname{grad}\left(P_{K} f\right) \cdot\left(\boldsymbol{x}-\boldsymbol{x}_{K}\right), v-\Pi v\right)_{K}+\left(f-P_{K} f, v-\Pi v\right)_{K}\right. \\
& \left.+\sum_{\gamma \subset \partial K}\left(\left(g-P_{\gamma} g, v-\Pi v\right)_{\gamma \cap \Gamma_{N}}-\left(\alpha_{\gamma}^{K} \frac{\partial}{\partial \hat{\boldsymbol{t}}_{\gamma}^{K}}\left[a \frac{\partial u_{n c}}{\partial \hat{\boldsymbol{n}}}\right]_{\gamma} s_{\gamma}^{K}, v\right)_{\gamma}\right)\right) .
\end{aligned}
$$




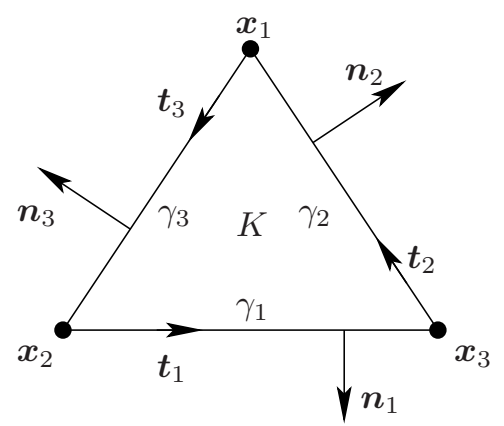

Figure 2. Direction and enumeration of the vertices, edges, tangent and normal vectors of element $K$.

The next step is to develop some convenient representations for the terms appearing on the right hand side. Fix an element $K \in \mathcal{P}$ and label the vertices, edges, tangents and normals as shown in Figure 2 ,

The following result will be used to deal with the first term in (3.5).

Lemma 3.1. Let $K \in \mathcal{P}$. Then, for all $v \in H_{E}^{1}(\Omega)$,

$$
\left(\operatorname{grad}\left(P_{K} f\right) \cdot\left(\boldsymbol{x}-\boldsymbol{x}_{K}\right), \Pi v\right)_{K}=-\frac{|K|}{10} \sum_{i=1}^{3}\left(\operatorname{grad}\left(P_{K} f\right) \cdot\left(\boldsymbol{x}_{i}-\boldsymbol{x}_{K}\right)\right) \bar{v}_{\gamma_{i}} .
$$

Proof. Observing that for $i=1,2,3,\left(\lambda_{i}, \theta_{K}\right)_{K}$ is independent of $i$ (due to symmetry of $\theta_{K}$ ), we see that

$$
\left(\lambda_{i}, \theta_{K}\right)_{K}=\frac{1}{3}\left(\theta_{K}, \sum_{j=1}^{3} \lambda_{j}\right)_{K}=\frac{1}{3}\left(\theta_{K}, 1\right)_{K}=\frac{|K|}{3}
$$

so $\left(\lambda_{i}-\frac{1}{3}, \theta_{K}\right)_{K}=0$. It then follows that

$$
\left(\operatorname{grad}\left(P_{K} f\right) \cdot\left(\boldsymbol{x}-\boldsymbol{x}_{K}\right), \theta_{K}\right)_{K}=0 .
$$

Moreover, for $\gamma_{i} \subset \partial K$, a simple calculation using (2.4) gives $\left(\lambda_{i}-\frac{1}{3}, \theta_{\gamma_{i}}\right)_{K}=$ $-\frac{1}{15}|K|$ and $\left(\lambda_{j}-\frac{1}{3}, \theta_{\gamma_{i}}\right)_{K}=\frac{1}{30}|K|$ if $i \neq j$ and hence, for $i=1,2,3$,

$$
\left(\operatorname{grad}\left(P_{K} f\right) \cdot\left(\boldsymbol{x}-\boldsymbol{x}_{K}\right), \theta_{\gamma_{i}}\right)_{K}=-\frac{|K|}{10}\left(\operatorname{grad}\left(P_{K} f\right) \cdot\left(\boldsymbol{x}_{i}-\boldsymbol{x}_{K}\right)\right) .
$$

The result then follows from (3.7) and (3.8) after inserting the definition (2.10) of $\Pi v$ into $\left(\operatorname{grad}\left(P_{K} f\right) \cdot\left(\boldsymbol{x}-\boldsymbol{x}_{K}\right), \Pi v\right)_{K}$.

The following identities will prove useful in what follows. For $i=1,2,3$, let $\boldsymbol{t}_{i}=\left|\gamma_{i}\right| \hat{\boldsymbol{t}}_{i}$ and $\boldsymbol{n}_{i}=\left|\gamma_{i}\right| \hat{\boldsymbol{n}}_{i}$. Then for $i=1,2,3$,

$$
\begin{gathered}
\left(\boldsymbol{t}_{i} \cdot \boldsymbol{n}_{i}\right)=0, \\
\left(\boldsymbol{t}_{1} \cdot \boldsymbol{n}_{2}\right)=\left(\boldsymbol{t}_{2} \cdot \boldsymbol{n}_{3}\right)=\left(\boldsymbol{t}_{3} \cdot \boldsymbol{n}_{1}\right)=2|K|, \\
\left(\boldsymbol{t}_{1} \cdot \boldsymbol{n}_{3}\right)=\left(\boldsymbol{t}_{2} \cdot \boldsymbol{n}_{1}\right)=\left(\boldsymbol{t}_{3} \cdot \boldsymbol{n}_{2}\right)=-2|K|,
\end{gathered}
$$


and

$$
\operatorname{grad} \lambda_{i}=-\frac{1}{2|K|} \boldsymbol{n}_{i}
$$

Lemma 3.2. Let $K \in \mathcal{P}$ be any element, and define the following functions on $K$ :

$$
\boldsymbol{\theta}_{1}^{K}=\alpha_{\gamma_{1}}^{K} \frac{\partial}{\partial \hat{\boldsymbol{t}}_{1}}\left[a \frac{\partial u_{n c}}{\partial \hat{\boldsymbol{n}}}\right]_{\gamma_{1}} \frac{\left|\gamma_{1}\right|^{2}}{4|K|}\left(\lambda_{2} \boldsymbol{t}_{3}+\lambda_{3} \boldsymbol{t}_{2}\right),
$$

with $\boldsymbol{\theta}_{2}^{K}$ and $\boldsymbol{\theta}_{3}^{K}$ defined analogously. Then for all $i, j=1,2,3$,

$$
\begin{gathered}
\left(\hat{\boldsymbol{n}}_{i} \cdot \boldsymbol{\theta}_{j}^{K}\right)_{\mid \gamma_{i}}=-\alpha_{\gamma_{i}}^{K} \frac{\partial}{\partial \hat{\boldsymbol{t}}_{i}}\left[a \frac{\partial u_{n c}}{\partial \hat{\boldsymbol{n}}}\right]_{\gamma_{i}} s_{\gamma_{i}}^{K} \delta_{i j}, \\
\operatorname{div} \boldsymbol{\theta}_{j}^{K}=0 \text { in } K,
\end{gathered}
$$

and there exists a positive constant $C$, depending only on the shape of the element, such that

$$
\left\|\boldsymbol{\theta}_{i}^{K}\right\|_{L_{2}(K)} \leq C h_{K}^{2} \alpha_{\gamma_{i}}^{K}\left|\frac{\partial}{\partial \hat{\boldsymbol{t}}_{i}}\left[a \frac{\partial u_{n c}}{\partial \hat{\boldsymbol{n}}}\right]_{\gamma_{i}}\right| .
$$

Proof. We shall prove these results for $\boldsymbol{\theta}_{1}^{K}$ with the results for $\boldsymbol{\theta}_{2}^{K}$ and $\boldsymbol{\theta}_{3}^{K}$ following by rotation. By (3.10) and (3.11),

$$
\begin{aligned}
\left(\hat{\boldsymbol{n}}_{1} \cdot \boldsymbol{\theta}_{1}^{K}\right) & =\alpha_{\gamma_{1}}^{K} \frac{\partial}{\partial \hat{\boldsymbol{t}}_{1}}\left[a \frac{\partial u_{n c}}{\partial \hat{\boldsymbol{n}}}\right]_{\gamma_{1}} \frac{\left|\gamma_{1}\right|^{2}}{4|K|}\left(\left(\hat{\boldsymbol{n}}_{1} \cdot \boldsymbol{t}_{3}\right) \lambda_{2}+\left(\hat{\boldsymbol{n}}_{1} \cdot \boldsymbol{t}_{2}\right) \lambda_{3}\right) \\
& =\alpha_{\gamma_{1}}^{K} \frac{\partial}{\partial \hat{\boldsymbol{t}}_{1}}\left[a \frac{\partial u_{n c}}{\partial \hat{\boldsymbol{n}}}\right]_{\gamma_{1}} \frac{\left|\gamma_{1}\right|}{2}\left(\lambda_{2}-\lambda_{3}\right)=-\alpha_{\gamma_{1}}^{K} \frac{\partial}{\partial \hat{\boldsymbol{t}}_{1}}\left[a \frac{\partial u_{n c}}{\partial \hat{\boldsymbol{n}}}\right]_{\gamma_{1}} s_{\gamma_{1}}^{K}
\end{aligned}
$$

where $s_{\gamma_{1}}^{K}$ denotes the arc length on $\gamma_{1}$. Upon applying (3.9) and observing that, for $i=1,2,3, \lambda_{i \mid \gamma_{i}}=0$ we obtain $\left(\hat{\boldsymbol{n}}_{2} \cdot \boldsymbol{\theta}_{1}^{K}\right)_{\mid \gamma_{2}}=0$ and $\left(\hat{\boldsymbol{n}}_{3} \cdot \boldsymbol{\theta}_{1}^{K}\right)_{\mid \gamma_{3}}=0$. Now, making use of (3.12) gives

$$
\operatorname{div} \boldsymbol{\theta}_{1}^{K}=-\alpha_{\gamma_{1}}^{K} \frac{\partial}{\partial \hat{\boldsymbol{t}}_{1}}\left[a \frac{\partial u_{n c}}{\partial \hat{\boldsymbol{n}}}\right]_{\gamma_{1}} \frac{\left|\gamma_{1}\right|^{2}}{8|K|^{2}}\left(\left(\boldsymbol{n}_{2} \cdot \boldsymbol{t}_{3}\right)+\left(\boldsymbol{n}_{3} \cdot \boldsymbol{t}_{2}\right)\right)
$$

and hence $\boldsymbol{\theta}_{1}^{K}$ is divergence free thanks to (3.10) and (3.11). Finally, (3.16) follows from standard scaling arguments after applying (2.4).

Lemma 3.3. Let $K \in \mathcal{P}$ be any element, and define the following function on $K$ :

$$
\begin{aligned}
\boldsymbol{\vartheta}^{K}= & \left(\operatorname{grad}\left(P_{K} f\right) \cdot\left(\boldsymbol{x}_{1}-\boldsymbol{x}_{K}\right)\right)\left(\frac{1}{20}-\frac{1}{3} \lambda_{1}\right)\left(\lambda_{2} \boldsymbol{t}_{3}-\lambda_{3} \boldsymbol{t}_{2}\right) \\
& +\left(\operatorname{grad}\left(P_{K} f\right) \cdot\left(\boldsymbol{x}_{2}-\boldsymbol{x}_{K}\right)\right)\left(\frac{1}{20}-\frac{1}{3} \lambda_{2}\right)\left(\lambda_{3} \boldsymbol{t}_{1}-\lambda_{1} \boldsymbol{t}_{3}\right) \\
& +\left(\operatorname{grad}\left(P_{K} f\right) \cdot\left(\boldsymbol{x}_{3}-\boldsymbol{x}_{K}\right)\right)\left(\frac{1}{20}-\frac{1}{3} \lambda_{3}\right)\left(\lambda_{1} \boldsymbol{t}_{2}-\lambda_{2} \boldsymbol{t}_{1}\right) .
\end{aligned}
$$

Then for $i=1,2,3$,

$$
\begin{gathered}
\left(\hat{\boldsymbol{n}}_{i} \cdot \boldsymbol{\vartheta}^{K}\right)_{\mid \gamma_{i}}=\frac{1}{10} \frac{|K|}{\left|\gamma_{i}\right|}\left(\operatorname{grad}\left(P_{K} f\right) \cdot\left(\boldsymbol{x}_{i}-\boldsymbol{x}_{K}\right)\right), \\
\operatorname{div} \boldsymbol{\vartheta}^{K}=\left(\operatorname{grad}\left(P_{K} f\right) \cdot\left(\boldsymbol{x}_{K}-\boldsymbol{x}\right)\right) \text { in } K,
\end{gathered}
$$


and there exists a positive constant $C$, depending only on the shape of the element, such that

$$
\left\|\vartheta^{K}\right\|_{L_{2}(K)} \leq C h_{K}^{3}\left|\operatorname{grad}\left(P_{K} f\right)\right| .
$$

Proof. Making use of the fact that $\lambda_{i}=0$ on $\gamma_{i}$ for $i=1,2,3$ and (3.9) we have

$$
\left(\hat{\boldsymbol{n}}_{1} \cdot \boldsymbol{\vartheta}^{K}\right)_{\mid \gamma_{1}}=\frac{1}{20}\left(\operatorname{grad}\left(P_{K} f\right) \cdot\left(\boldsymbol{x}_{1}-\boldsymbol{x}_{K}\right)\right)\left(\left(\hat{\boldsymbol{n}}_{1} \cdot \boldsymbol{t}_{3}\right) \lambda_{2}-\left(\hat{\boldsymbol{n}}_{1} \cdot \boldsymbol{t}_{2}\right) \lambda_{3}\right) .
$$

Applying (3.10) and (3.11) yields $\left(\hat{\boldsymbol{n}}_{1} \cdot \boldsymbol{\vartheta}^{K}\right)_{\mid \gamma_{1}}=\frac{1}{10} \frac{|K|}{\left|\gamma_{1}\right|}\left(\operatorname{grad}\left(P_{K} f\right) \cdot\left(\boldsymbol{x}_{1}-\boldsymbol{x}_{K}\right)\right)$ upon noting that $\lambda_{2}+\lambda_{3}=1$ on $\gamma_{1}$. The remaining terms in (3.18) follow by symmetry. To show (3.19) we can apply the product rule, (3.12), (3.10) and (3.11) to obtain

$$
\begin{aligned}
\operatorname{div} \boldsymbol{\vartheta}^{K}= & \frac{1}{3} \operatorname{grad}\left(P_{K} f\right) \cdot\left(\left(\boldsymbol{x}_{2}+\boldsymbol{x}_{3}-2 \boldsymbol{x}_{1}\right) \lambda_{1}+\left(\boldsymbol{x}_{1}+\boldsymbol{x}_{3}-2 \boldsymbol{x}_{2}\right) \lambda_{2}\right. \\
& \left.+\left(\boldsymbol{x}_{1}+\boldsymbol{x}_{2}-2 \boldsymbol{x}_{3}\right) \lambda_{3}\right)=\left(\operatorname{grad}\left(P_{K} f\right) \cdot\left(\boldsymbol{x}_{K}-\boldsymbol{x}\right)\right) .
\end{aligned}
$$

Also, (2.4) and standard scaling arguments can be combined to arrive at (3.20).

Lemma 3.4. Let $\boldsymbol{\sigma}_{K}=\boldsymbol{\vartheta}^{K}+\sum_{i=1}^{3} \boldsymbol{\theta}_{i}^{K}$ where $\boldsymbol{\theta}_{i}^{K}$ and $\boldsymbol{\vartheta}^{K}$ are defined in Lemma 3.2 and Lemma 3.3 respectively. Then

$$
\begin{gathered}
\left(\boldsymbol{\sigma}_{K}, \operatorname{grad} v\right)_{K}=\left(\operatorname{grad}\left(P_{K} f\right) \cdot\left(\boldsymbol{x}-\boldsymbol{x}_{K}\right), v-\Pi v\right)_{K} \\
-\sum_{\gamma \subset \partial K}\left(\alpha_{\gamma}^{K} \frac{\partial}{\partial \hat{\boldsymbol{t}}_{\gamma}^{K}}\left[a \frac{\partial u_{n c}}{\partial \hat{\boldsymbol{n}}}\right]_{\gamma} s_{\gamma}^{K}, v\right)_{\gamma} .
\end{gathered}
$$

Proof. By Lemma 3.1 we have that

$$
\begin{aligned}
& \left(\operatorname{grad}\left(P_{K} f\right) \cdot\left(\boldsymbol{x}-\boldsymbol{x}_{K}\right), v-\Pi v\right)_{K} \\
& \quad-\sum_{\gamma \subset \partial K}\left(\alpha_{\gamma}^{K} \frac{\partial}{\partial \hat{\boldsymbol{t}}_{\gamma}^{K}}\left[a \frac{\partial u_{n c}}{\partial \hat{\boldsymbol{n}}}\right]_{\gamma} s_{\gamma}^{K}, v\right)_{\gamma}=\left(\operatorname{grad}\left(P_{K} f\right) \cdot\left(\boldsymbol{x}-\boldsymbol{x}_{K}\right), v\right)_{K} \\
& \quad+\sum_{i=1}^{3}\left(\frac{1}{10} \frac{|K|}{\left|\gamma_{i}\right|}\left(\operatorname{grad}\left(P_{K} f\right) \cdot\left(\boldsymbol{x}_{i}-\boldsymbol{x}_{K}\right)\right)-\alpha_{\gamma_{i}}^{K} \frac{\partial}{\partial \hat{\boldsymbol{t}}_{i}}\left[a \frac{\partial u_{n c}}{\partial \hat{\boldsymbol{n}}}\right]_{\gamma_{i}} s_{\gamma_{i}}^{K}, v\right)_{\gamma_{i}} .
\end{aligned}
$$

Also, using integration by parts gives

$$
\left(\boldsymbol{\sigma}_{K}, \operatorname{grad} v\right)_{K}=\sum_{i=1}^{3}\left(\hat{\boldsymbol{n}}_{i} \cdot \boldsymbol{\sigma}_{K}, v\right)_{\gamma_{i}}-\left(\operatorname{div} \boldsymbol{\sigma}_{K}, v\right)_{K} \cdot
$$

So the result is true if for $i=1,2,3$,

$$
\hat{\boldsymbol{n}}_{i} \cdot \boldsymbol{\sigma}_{K}=\frac{1}{10} \frac{|K|}{\left|\gamma_{i}\right|}\left(\operatorname{grad}\left(P_{K} f\right) \cdot\left(\boldsymbol{x}_{i}-\boldsymbol{x}_{K}\right)\right)-\alpha_{\gamma_{i}}^{K} \frac{\partial}{\partial \hat{\boldsymbol{t}}_{i}}\left[a \frac{\partial u_{n c}}{\partial \hat{\boldsymbol{n}}}\right]_{\gamma_{i}} s_{\gamma_{i}}^{K}
$$

and $-\operatorname{div} \boldsymbol{\sigma}_{K}=\operatorname{grad}\left(P_{K} f\right) \cdot\left(\boldsymbol{x}-\boldsymbol{x}_{K}\right)$ which follows by applying Lemma 3.2 and Lemma 3.3 to the expression for $\boldsymbol{\sigma}_{K}$.

We are now in a position to prove our upper bound for $\|\phi\|$. 
Lemma 3.5. For each $K \in \mathcal{P}$, let $\beta_{K} \in H_{0}^{1}(K)$ be given and define

$$
\begin{aligned}
\Phi_{K}\left(\beta_{K}\right)=a_{K}^{-1 / 2}\left(\left\|\sigma_{K}^{\star}\right\|_{L_{2}(K)}\right. & +C_{K}\left\|f-P_{K} f\right\|_{L_{2}(K)} \\
& \left.+\sum_{\gamma \subset \partial K \cap \Gamma_{N}} C_{\gamma}^{K}\left\|g-P_{\gamma} g\right\|_{L_{2}(\gamma)}\right)
\end{aligned}
$$

where $\boldsymbol{\sigma}_{K}^{\star}=\boldsymbol{\sigma}_{K}-\operatorname{curl} \beta_{K}$. Then

$$
\|\phi\|^{2} \leq \sum_{K \in \mathcal{P}} \Phi_{K}^{2}\left(\beta_{K}\right) .
$$

Proof. Returning to (3.5) and using Lemma 3.4 we obtain

$$
\begin{aligned}
(a \operatorname{grad} \phi, \operatorname{grad} v)= & \sum_{K \in \mathcal{P}}\left(\left(\boldsymbol{\sigma}_{K}, \operatorname{grad} v\right)_{K}+\left(f-P_{K} f, v-\Pi v\right)_{K}\right. \\
& \left.+\sum_{\gamma \subset \partial K \cap \Gamma_{N}}\left(g-P_{\gamma} g, v-\Pi v\right)_{\gamma}\right) .
\end{aligned}
$$

Since for all $K \in \mathcal{P}$ and $\gamma \subset \partial K,\left(\hat{\boldsymbol{n}}_{\gamma}^{K} \cdot \operatorname{curl} \beta_{K}\right)=0$ and $\operatorname{div}\left(\operatorname{curl} \beta_{K}\right)=0$,

$$
\left(\boldsymbol{\sigma}_{K}, \operatorname{grad} v\right)_{K}=\left(\boldsymbol{\sigma}_{K}^{\star}, \operatorname{grad} v\right)_{K} .
$$

Inserting (3.25) into (3.24) then applying Cauchy-Schwarz followed by inequalities (2.13) and (2.15) yields

$$
(a \operatorname{grad} \phi, \operatorname{grad} v) \leq \sum_{K \in \mathcal{P}} \Phi_{K}\left(\beta_{K}\right)\|v\|_{K} \leq\left(\sum_{K \in \mathcal{P}} \Phi_{K}^{2}\left(\beta_{K}\right)\right)^{1 / 2}\|v\|
$$

from which we can obtain the result by letting $v=\phi$ and dividing both sides of the inequality by $\|\phi\|$.

This result enables us to obtain computable bounds on $\|\phi\|$ for any choice of $\beta_{K}$. The simplest choice is $\beta_{K}=0$, however, by minimising over $\beta_{K}$ from an appropriate class of bubble function we obtain $\left\|\boldsymbol{\sigma}_{K}^{\star}\right\|_{L_{2}(K)} \leq\left\|\boldsymbol{\sigma}_{K}\right\|_{L_{2}(K)}$ and thereby potentially tighten the bound. We shall elaborate on this point later in the final section.

3.2. Local lower bounds. We now show that $\Phi_{K}$ also provides a lower bound for the conforming component of the error plus data oscillation up to a generic constant. In principle, one could obtain numerical estimates for the constant using techniques similar to those in [18. We shall not pursue this further here. Observing that as before we obtain $\left\|\boldsymbol{\sigma}_{K}^{\star}\right\|_{L_{2}(K)} \leq\left\|\boldsymbol{\sigma}_{K}\right\|_{L_{2}(K)}$, then applying the estimates (3.16) and (3.20) to the expression for $\boldsymbol{\sigma}_{K}$ and the estimates $C_{K} \leq C|K|^{1 / 2}$ and $C_{\gamma}^{K} \leq C|\gamma|^{1 / 2}$ to the expression for $\Phi_{K}\left(\beta_{K}\right)$ allows us to say that

$$
\begin{aligned}
\Phi_{K}\left(\beta_{K}\right) \leq & C a_{K}^{-1 / 2}\left(h_{K}^{3}\left|\operatorname{grad}\left(P_{K} f\right)\right|+\operatorname{osc}(f, K)\right. \\
& \left.+\sum_{\gamma \subset \partial K}\left(h_{K}^{2} \alpha_{\gamma}^{K}\left|\frac{\partial}{\partial \hat{\boldsymbol{t}}_{\gamma}^{K}}\left[a \frac{\partial u_{n c}}{\partial \hat{\boldsymbol{n}}}\right]_{\gamma}\right|+\operatorname{osc}\left(g, \gamma \cap \Gamma_{N}\right)\right)\right) .
\end{aligned}
$$

In the next result we obtain an estimate for $\left|\operatorname{grad}\left(P_{K} f\right)\right|$. 
Lemma 3.6. There exists a positive constant $C$, depending only on the shape of the element, such that

$$
a_{K}^{-1 / 2} h_{K}^{3}\left|\operatorname{grad}\left(P_{K} f\right)\right| \leq C\left(\|\phi\|_{K}+a_{K}^{-1 / 2} \operatorname{osc}(f, K)\right) .
$$

Proof. Let $\vec{x}_{K} \in \mathbb{R}^{3}$ and $\vec{y}_{K} \in \mathbb{R}^{3}$ denote the vectors formed from the first and second rows of the matrix $\boldsymbol{M}=\left[\begin{array}{lll}\boldsymbol{x}_{1}-\boldsymbol{x}_{K} & \boldsymbol{x}_{2}-\boldsymbol{x}_{K} & \boldsymbol{x}_{3}-\boldsymbol{x}_{K}\end{array}\right]$ and let the function $\boldsymbol{v}^{K}$ take the value $\left(\boldsymbol{x}-\boldsymbol{x}_{K}\right) \lambda_{1} \lambda_{2} \lambda_{3}$ on $K$ and be equal to zero everywhere else. Computations using the definition of $\Pi$ and (2.4) give us that $\Pi \boldsymbol{v}^{K}=\mathbf{0}$, $\left(x-x_{K}, \boldsymbol{v}^{K}\right)_{K}=\frac{|K|}{1260}\left(\begin{array}{c}\left|\vec{x}_{K}\right|^{2} \\ \left(\vec{x}_{K} \cdot \vec{y}_{K}\right)\end{array}\right)$ and $\left(y-y_{K}, \boldsymbol{v}^{K}\right)_{K}=\frac{|K|}{1260}\left(\begin{array}{c}\left(\vec{x}_{K} \cdot \vec{y}_{K}\right) \\ \left|\vec{y}_{K}\right|^{2}\end{array}\right)$, where $\Pi$ applied to a vector-valued function is defined as $\Pi$ applied to each component. Using these equalities and the fact that $\boldsymbol{v}^{K}=\mathbf{0}$ on all edges $\gamma \subset \partial \mathcal{P}$, we can choose $v$ to be each component of $\boldsymbol{v}^{K}$ in (3.5) to obtain

$$
\begin{aligned}
& \frac{|K|}{1260}\left[\begin{array}{cc}
\left|\vec{x}_{K}\right|^{2} & \left(\vec{x}_{K} \cdot \vec{y}_{K}\right) \\
\left(\vec{x}_{K} \cdot \vec{y}_{K}\right) & \left|\vec{y}_{K}\right|^{2}
\end{array}\right] \operatorname{grad}\left(P_{K} f\right) \\
& \quad=\left(a_{K} \operatorname{grad} \phi, \operatorname{grad} \boldsymbol{v}^{K}\right)_{K}-\left(f-P_{K} f, \boldsymbol{v}^{K}\right)_{K} .
\end{aligned}
$$

It is not difficult to see that

$$
\left|\vec{x}_{K}\right|^{2}\left|\vec{y}_{K}\right|^{2}-\left(\vec{x}_{K} \cdot \vec{y}_{K}\right)^{2}=\left|\vec{x}_{K} \times \vec{y}_{K}\right|^{2}=\sum_{i=1}^{3}\left(\operatorname{det}\left(\boldsymbol{M}_{i}\right)\right)^{2},
$$

where $\boldsymbol{M}_{i}$ is the $2 \times 2$ sub-matrix of $\boldsymbol{M}$ obtained by omitting the $i$-th column. Then, we note that $\operatorname{det}\left(\boldsymbol{M}_{i}\right)= \pm 2\left|K_{i}\right|$, where $K_{i}$ is the triangle formed by the endpoints of $\gamma_{i}$ and the centroid of $K$. Hence,

$$
\left|\vec{x}_{K}\right|^{2}\left|\vec{y}_{K}\right|^{2}-\left(\vec{x}_{K} \cdot \vec{y}_{K}\right)^{2}=4 \sum_{i=1}^{3}\left|K_{i}\right|^{2} \geq \frac{4}{3}|K|^{2} .
$$

This implies that the matrix on the left hand side of (3.28) is non-singular and can be inverted to give an expression for grad $\left(P_{K} f\right)$. Now, applying Cauchy-Schwarz to this expression and observing that $\left\|\boldsymbol{v}^{K}\right\|_{K} \leq C a_{K}^{1 / 2} h_{K}$ and that $\left|\vec{x}_{K}\right|^{2},\left|\vec{y}_{K}\right|^{2}$, $\left(\vec{x}_{K} \cdot \vec{y}_{K}\right)$ and $\left\|\boldsymbol{v}^{K}\right\|_{L_{2}(K)}$ are all bounded by $C h_{K}^{2}$, we have that

$$
\left|\operatorname{grad}\left(P_{K} f\right)\right| \leq C h_{K}^{-3}\left(a_{K}^{1 / 2}\|\phi\|_{K}+\operatorname{osc}(f, K)\right)
$$

from which we can obtain the result claimed.

We now work to obtain an estimate for $\alpha_{\gamma}^{K}\left|\frac{\partial}{\partial \hat{\boldsymbol{t}}_{\gamma}^{K}}\left[a \frac{\partial u_{n c}}{\partial \tilde{\boldsymbol{n}}}\right]_{\gamma}\right|$. To this end, the following result will be useful.

Lemma 3.7. Let $K \in \mathcal{P}$ and $\gamma \subset \partial K$. Define $\beta_{\gamma}^{K}=60 \lambda_{l} \lambda_{r}\left(\lambda_{r}-\lambda_{l}\right)$ where $\mathcal{V}(\gamma)=\{l, r\}$ and the edge $\gamma$ is oriented as in Figure 1 . Then for $p \in \mathbb{P}_{1}(\gamma)$,

$$
\left(p, \beta_{\gamma}^{K}\right)_{\gamma}=\frac{\partial p}{\partial \hat{\boldsymbol{t}}_{\gamma}^{K}}|\gamma|^{2} .
$$

Proof. Letting $p=r_{1} s_{\gamma}^{K}+r_{2}$, where $r_{1}, r_{2} \in \mathbb{R}$, and using (2.6) then (2.5) gives $\left(p, \beta_{\gamma}^{K}\right)_{\gamma}=r_{1}|\gamma|^{2}$. So the result holds by applying (2.7) to show that $\frac{\partial p}{\partial \hat{\boldsymbol{t}}_{\gamma}^{K}}=r_{1}$. 
Lemma 3.8. Let $\gamma \subset \partial \mathcal{P} \backslash \Gamma_{D}$. Then there exists a positive constant $C$, depending only on the shape of the elements, such that

$$
\begin{aligned}
& a_{K}^{-\frac{1}{2}} h_{K}^{2} \alpha_{\gamma}^{K}\left|\frac{\partial}{\partial \hat{\boldsymbol{t}}_{\gamma}^{K}}\left[a \frac{\partial u_{n c}}{\partial \hat{\boldsymbol{n}}}\right]_{\gamma}\right| \\
& \leq C \begin{cases}\|\phi\|_{K}+\|\phi\|_{K^{\prime}}+a_{K}^{-\frac{1}{2}}\left(\operatorname{osc}(f, K)+\operatorname{osc}\left(f, K^{\prime}\right)\right) & \text { if } \gamma=\partial K \cap \partial K^{\prime}, \\
\|\phi\|_{K}+a_{K}^{-\frac{1}{2}}(\operatorname{osc}(f, K)+\operatorname{osc}(g, \gamma)) & \text { if } \gamma=\partial K \cap \Gamma_{N} .\end{cases}
\end{aligned}
$$

Proof. Let $\gamma$ be an interior edge and observe that

$$
h_{K}^{2}\left|\frac{\partial}{\partial \hat{\boldsymbol{t}}_{\gamma}^{K}}\left[a \frac{\partial u_{n c}}{\partial \hat{\boldsymbol{n}}}\right]_{\gamma}\right| \leq C|\gamma|^{2}\left|\frac{\partial}{\partial \hat{\boldsymbol{t}}_{\gamma}}\left[a \frac{\partial u_{n c}}{\partial \hat{\boldsymbol{n}}}\right]_{\gamma}\right|
$$

where $\hat{\boldsymbol{t}}_{\gamma}$ is either of the two tangents to $\gamma$. We define $v_{\gamma} \in H_{E}^{1}(\Omega)$ by the rule $v_{\gamma}=60 \lambda_{l} \lambda_{r}\left(\lambda_{r}-\lambda_{l}\right)$ where $\mathcal{V}(\gamma)=\{l, r\}$. Using (2.4) we have that $\left(v_{\gamma}, 1\right)_{K}=0$ for all $K \in \mathcal{P}$ and $\left(v_{\gamma}, 1\right)_{\gamma^{\prime}}=0$ for all $\gamma^{\prime} \subset \partial \mathcal{P}$ which together imply that $\Pi v_{\gamma}=0$. Using these equalities, the fact that $v_{\gamma}$ is zero on all edges except $\gamma$ and Lemma 3.7. and by letting $v=v_{\gamma}$ in (3.5) we obtain

$$
\begin{aligned}
|\gamma|^{2}\left|\frac{\partial}{\partial \hat{\boldsymbol{t}}_{\gamma}}\left[a \frac{\partial u_{n c}}{\partial \hat{\boldsymbol{n}}}\right]_{\gamma}\right|= & \mid \sum_{\substack{K \in \mathcal{P}_{\dot{K}} \\
\gamma \subset \partial K}}\left(-\left(a \operatorname{grad} \phi, \operatorname{grad} v_{\gamma}\right)_{K}\right. \\
& \left.+\left(\operatorname{grad}\left(P_{K} f\right) \cdot\left(\boldsymbol{x}-\boldsymbol{x}_{K}\right), v_{\gamma}\right)_{K}+\left(f-P_{K} f, v_{\gamma}\right)_{K}\right) \mid .
\end{aligned}
$$

Now, making use of Cauchy-Schwarz, the definition of the oscillation of $f$ and the estimates $\left\|\operatorname{grad}\left(P_{K} f\right) \cdot\left(\boldsymbol{x}-\boldsymbol{x}_{K}\right)\right\|_{L_{2}(K)} \leq C h_{K}^{2}\left|\operatorname{grad}\left(P_{K} f\right)\right|,\left\|v_{\gamma}\right\|_{K} \leq C a_{K}^{1 / 2}$ and $\left\|v_{\gamma}\right\|_{L_{2}(K)} \leq C h_{K}$ we have that

$$
|\gamma|^{2}\left|\frac{\partial}{\partial \hat{\boldsymbol{t}}_{\gamma}}\left[a \frac{\partial u_{n c}}{\partial \hat{\boldsymbol{n}}}\right]_{\gamma}\right| \leq C \sum_{\substack{K \in \mathcal{P}_{\dot{K}} \\ \gamma \subset \partial K}}\left(a_{K}^{1 / 2}\|\phi\|_{K}+\operatorname{osc}(f, K)+h_{K}^{3}\left|\operatorname{grad}\left(P_{K} f\right)\right|\right)
$$

to which we can apply Lemma 3.6 to get

$$
|\gamma|^{2}\left|\frac{\partial}{\partial \hat{\boldsymbol{t}}_{\boldsymbol{\gamma}}}\left[a \frac{\partial u_{n c}}{\partial \hat{\boldsymbol{n}}}\right]_{\gamma}\right| \leq C \sum_{\substack{K \in \mathcal{P}_{\mathcal{B}} \\ \gamma \subset \partial K}}\left(a_{K}^{1 / 2}\|\phi\|_{K}+\operatorname{osc}(f, K)\right) .
$$

Finally, by letting $\gamma$ be a common edge of elements $K$ and $K^{\prime}$ and using the definition of $\alpha_{\gamma}^{K}$, we have that

$$
\begin{aligned}
& a_{K}^{-\frac{1}{2}} h_{K}^{2} \alpha_{\gamma}^{K}\left|\frac{\partial}{\partial \hat{\boldsymbol{t}}_{\gamma}}\left[a \frac{\partial u_{n c}}{\partial \hat{\boldsymbol{n}}}\right]_{\gamma}\right| \\
& \quad \leq C\left(a_{K}^{-\frac{1}{2}} \alpha_{\gamma}^{K}\left(\left(a_{K}^{1 / 2}+a_{K^{\prime}}^{1 / 2}\right)\left(\|\phi\|_{K}+\|\phi\|_{K^{\prime}}\right)+\operatorname{osc}(f, K)+\operatorname{osc}\left(f, K^{\prime}\right)\right)\right) \\
& \quad=C\left(\|\phi\|_{K}+\|\phi\|_{K^{\prime}}+a_{K}^{-\frac{1}{2}} \alpha_{\gamma}^{K}\left(\operatorname{osc}(f, K)+\operatorname{osc}\left(f, K^{\prime}\right)\right)\right),
\end{aligned}
$$

and the estimate follows since $\alpha_{\gamma}^{K} \leq 1$. The result for $\gamma=\partial K \cap \Gamma_{N}$ can be proved similarly.

Finally, collecting these results we obtain the following lemma. 
Lemma 3.9. Let $\hat{K}$ be the set of elements, including element $K$, that share an edge with element $K$. Then there exists a positive constant $c$, depending only on the shape of the elements, such that

$$
c \Phi_{K}\left(\beta_{K}\right) \leq \sum_{K^{\prime} \subset \hat{K}}\left(\|\phi\|_{K^{\prime}}+a_{K}^{-1 / 2} \operatorname{osc}\left(f, K^{\prime}\right)\right)+a_{K}^{-1 / 2} \sum_{\gamma \subset \partial K \cap \Gamma_{N}} \operatorname{osc}(g, \gamma) .
$$

Proof. Inserting the estimates proved in Lemma 3.6 and Lemma 3.8 into (3.26) gives the result.

\section{Estimation OF THE NONCONFORMING ERROR}

The main part of the upper bound for $\left(a^{-1} \operatorname{curl} \psi, \operatorname{curl} \psi\right)$ is defined analogously to [1] by the continuous piecewise quadratic interpolant of $u_{n c}$ on $\mathcal{P}$ whose values at the nodes are given by

$$
\mathcal{S}\left(u_{n c}\right)\left(\boldsymbol{x}_{n}\right)=\left\{\begin{array}{l}
q\left(\boldsymbol{x}_{n}\right) \text { if } n \in \mathcal{N}\left(\Gamma_{D}\right), \\
\sum_{K \in \Omega_{n}} \omega_{K, n} u_{n c \mid K}\left(\boldsymbol{x}_{n}\right) \text { if } n \notin \mathcal{N}\left(\Gamma_{D}\right)
\end{array}\right.
$$

for $n \in \mathcal{N}$ with the weights $\omega_{K, n}$ defined by $\omega_{K, n}=a_{K}^{1 / 2} / \sum_{K^{\prime} \subset \Omega_{n}} a_{K^{\prime}}^{1 / 2}$. As in [1], it is essential that a weighted average be used in order that robustness of the estimator with respect to the permeability coefficient is maintained. Note that these weights satisfy the condition

$$
\sum_{K \subset \Omega_{n}} \omega_{K, n}=1
$$

The following result can easily be obtained from the upper bound proved in 1 and so we omit the proof.

Lemma 4.1. Let

$$
\Psi_{K}=\left\|u_{n c}-\mathcal{S}\left(u_{n c}\right)\right\|_{K}+\sum_{\gamma \subset \partial K \cap \Gamma_{D}} a_{K}^{1 / 2} \operatorname{osc}(q, \gamma)
$$

Then

$$
\left(a^{-1} \operatorname{curl} \psi, \operatorname{curl} \psi\right) \leq \sum_{K \in \mathcal{P}} \Psi_{K}^{2} .
$$

4.1. Local lower bounds. By analogy with [1] we shall require the following lemma in order to prove that $\Psi_{K}$ also provides a lower bound for the nonconforming component of the error plus data oscillation.

Lemma 4.2. Let $n \in \mathcal{N}$, let $K \in \Omega_{n}$ and let $\partial \Omega_{n}$ denote the boundary of $\Omega_{n}$. Then there exists a positive constant $C$, depending only on the shape of the elements, such that

$$
\begin{aligned}
& \text { (4.5) }\left|u_{n c \mid K}\left(\boldsymbol{x}_{n}\right)-\mathcal{S}\left(u_{n c}\right)\left(\boldsymbol{x}_{n}\right)\right| \\
& \leq C\left\{\begin{array}{c}
\left(a^{-1} \operatorname{curl} \psi, \operatorname{curl} \psi\right)_{\Omega_{n}}^{1 / 2} \sum_{K^{\prime} \subset \Omega_{n} \backslash K} \omega_{K^{\prime}, n} a_{K K^{\prime}}^{-1 / 2} \text { if } n \notin \mathcal{N}\left(\Gamma_{D}\right), \\
a_{K \Gamma_{D}, n}^{-1 / 2}\left(a^{-1} \operatorname{curl} \psi, \operatorname{curl} \psi\right)_{\Omega_{n}}^{1 / 2}+\sum_{\gamma \subset \partial \Omega_{n} \cap \Gamma_{D}} \operatorname{osc}(q, \gamma) \text { if } n \in \mathcal{N}\left(\Gamma_{D}\right) .
\end{array}\right.
\end{aligned}
$$


Proof. First let $n \notin \mathcal{N}\left(\Gamma_{D}\right)$. Inserting definition (4.1) and using property (4.2), we obtain

$$
u_{n c \mid K}\left(\boldsymbol{x}_{n}\right)-\mathcal{S}\left(u_{n c}\right)\left(\boldsymbol{x}_{n}\right)=\sum_{K^{\prime} \subset \Omega_{n} \backslash K} \omega_{K^{\prime}, n}\left(u_{n c \mid K}\left(\boldsymbol{x}_{n}\right)-u_{n c \mid K^{\prime}}\left(\boldsymbol{x}_{n}\right)\right) .
$$

For $n \in \mathcal{N}$ if $\boldsymbol{x}_{n} \notin \overline{\Gamma_{D}}$ is a vertex or edge midpoint of one element $K$ only, then Lemma 4.2 and (4.6) both imply that $\left|u_{n c \mid K}\left(\boldsymbol{x}_{n}\right)-\mathcal{S}\left(u_{n c}\right)\left(\boldsymbol{x}_{n}\right)\right| \leq 0$. It therefore suffices to consider the case when $\boldsymbol{x}_{n}$ is a common vertex or edge midpoint of more than one element. We first consider the contribution to (4.6) arising when an element $K^{\prime}$ shares a common edge $\gamma$ with element $K$.

On $\gamma=\partial K \cap \partial K^{\prime}$, as noted following (2.2), $\left[u_{n c}\right]_{\gamma}$ vanishes at the two GaussLegendre points on $\gamma$, so $u_{n c \mid K}-u_{n c \mid K^{\prime}}=r\left(\left(s_{\gamma}^{K}\right)^{2}-\frac{1}{12}|\gamma|^{2}\right)$ for a constant $r \in \mathbb{R}$. Hence, forming second derivatives, we obtain $2 r=\frac{\partial}{\partial \hat{\boldsymbol{t}}_{\gamma}^{K}}\left[\frac{\partial u_{n c}}{\partial \hat{\boldsymbol{t}}}\right]_{\gamma}$ where $\left[\frac{\partial u_{n c}}{\partial \hat{\boldsymbol{t}}}\right]_{\gamma}=$ $\frac{\partial u_{n c \mid K}}{\partial \hat{\boldsymbol{t}}_{\gamma}^{K}}+\frac{\partial u_{n c \mid K^{\prime}}}{\partial \hat{\boldsymbol{t}}_{\gamma}^{K^{\prime}}}$. So, letting $s_{\gamma}^{K}= \pm \frac{|\gamma|}{2}$ we obtain for a vertex $\boldsymbol{x}_{n} \in \gamma$,

$$
u_{n c \mid K}\left(\boldsymbol{x}_{n}\right)-u_{n c \mid K^{\prime}}\left(\boldsymbol{x}_{n}\right)=\mu|\gamma|^{2} \frac{\partial}{\partial \hat{\boldsymbol{t}}_{\gamma}^{K}}\left[\frac{\partial u_{n c}}{\partial \hat{\boldsymbol{t}}}\right]_{\gamma}
$$

where $\mu=\frac{1}{12}$, while taking $s_{\gamma}^{K}=0$ gives a similar identity for the midpoint $\boldsymbol{x}_{n} \in \gamma$, namely (4.7) with $\mu=-\frac{1}{24}$. Let the function $\beta_{\gamma} \in \mathcal{H}$ take the value $\mu \beta_{\gamma}^{K}$ on $K$, $-\mu \beta_{\gamma}^{K^{\prime}}$ on $K^{\prime}$ and zero everywhere else with the function $\beta_{\gamma}^{K}$ having been defined in Lemma 3.7. Now letting $w=\beta_{\gamma}$ in (2.21) and integrating the right hand side of the resulting expression by parts gives

$$
\left(a^{-1} \operatorname{curl} \psi, \operatorname{curl} \beta_{\gamma}\right)_{K \cup K^{\prime}}=\left(\beta_{\gamma},\left[\frac{\partial u_{n c}}{\partial \hat{\boldsymbol{t}}}\right]_{\gamma}\right)_{\gamma}=\mu|\gamma|^{2} \frac{\partial}{\partial \hat{\boldsymbol{t}}_{\gamma}^{K}}\left[\frac{\partial u_{n c}}{\partial \hat{\boldsymbol{t}}}\right]_{\gamma}
$$

by applying Lemma 3.7. Combining this with (4.7) yields

$$
u_{n c \mid K}\left(\boldsymbol{x}_{n}\right)-u_{n c \mid K^{\prime}}\left(\boldsymbol{x}_{n}\right)=\left(a^{-1} \operatorname{curl} \psi, \operatorname{curl} \beta_{\gamma}\right)_{K \cup K^{\prime}} .
$$

This relation is valid for pairs of elements $K$ and $K^{\prime}$ sharing a common edge $\gamma$. More generally, suppose elements $K$ and $K^{\prime}$ share only a common vertex $\boldsymbol{x}_{n}$. The path $\wp^{*}\left(K, K^{\prime}\right)$ appearing in (2.17) links the elements $K$ and $K^{\prime}$ by a set of elements having a common vertex at $\boldsymbol{x}_{n}$. The set of edges shared by these elements is denoted by $\partial \wp^{*}\left(K, K^{\prime}\right)$. Relation (4.8) holds on each edge along the path, and so, by summing (4.8) over edges, we obtain a telescoping sum of differences of $u_{n c}$ across neighbouring edges, which simplifies to give

$$
u_{n c \mid K}\left(\boldsymbol{x}_{n}\right)-u_{n c \mid K^{\prime}}\left(\boldsymbol{x}_{n}\right)=\left(a^{-1} \operatorname{curl} \psi, \operatorname{curl} \beta_{K K^{\prime}}\right)_{\Omega_{n}}
$$

where $\beta_{K K^{\prime}}=\sum_{\gamma^{\prime} \subset \wp_{\wp}{ }^{*}\left(K, K^{\prime}\right)} \beta_{\gamma^{\prime}}$. Applying Cauchy-Schwarz followed by the estimate $\left\|a^{-1 / 2} \operatorname{curl} \beta_{K K^{\prime}}\right\|_{L_{2}\left(\Omega_{n}\right)} \leq C a_{K K^{\prime}}^{-1 / 2}$ gives

$$
\left|u_{n c \mid K}\left(\boldsymbol{x}_{n}\right)-u_{n c \mid K^{\prime}}\left(\boldsymbol{x}_{n}\right)\right| \leq C a_{K K^{\prime}}^{-1 / 2}\left(a^{-1} \operatorname{curl} \psi, \operatorname{curl} \psi\right)_{\Omega_{n}}^{1 / 2} .
$$

The result is then obtained by inserting this estimate into the expression obtained by applying the triangle inequality to (4.6) after taking its modulus. 
Now, let $\boldsymbol{x}_{n}$ be an endpoint or midpoint of an edge $\gamma \subset \Gamma_{D} \cap \partial K^{\prime}$, where we allow for the possibility that $K^{\prime}=K$, with $K^{\prime}$ chosen so that it maximises $a_{K K^{\prime}}$ and observe that

$$
u_{n c \mid K}\left(\boldsymbol{x}_{n}\right)-\mathcal{S}\left(u_{n c}\right)\left(\boldsymbol{x}_{n}\right)=u_{n c \mid K}\left(\boldsymbol{x}_{n}\right)-u_{n c \mid K^{\prime}}\left(\boldsymbol{x}_{n}\right)+u_{n c \mid K^{\prime}}\left(\boldsymbol{x}_{n}\right)-q\left(\boldsymbol{x}_{n}\right) .
$$

Recalling that $q\left(\boldsymbol{x}_{n}\right)=q_{I}\left(\boldsymbol{x}_{n}\right), u_{n c \mid K^{\prime}}-q_{I}$ vanishes at the two Gauss-Legendre points on $\gamma$ and applying Lemma 3.7 allows us to write

$$
u_{n c \mid K^{\prime}}\left(\boldsymbol{x}_{n}\right)-q\left(\boldsymbol{x}_{n}\right)=\mu|\gamma|^{2} \frac{\partial}{\partial \hat{\boldsymbol{t}}_{\gamma}^{K^{\prime}}}\left(\frac{\partial u_{n c \mid K^{\prime}}}{\partial \hat{\boldsymbol{t}}_{\gamma}^{K^{\prime}}}\right)-\left(\beta_{\gamma}, \frac{\partial q_{I}}{\partial \hat{\boldsymbol{t}}_{\gamma}^{K^{\prime}}}\right)_{\gamma}
$$

where $\beta_{\gamma}=\mu \beta_{\gamma}^{K^{\prime}}$ on $K^{\prime}$ and zero elsewhere. As before letting $w=\beta_{\gamma}$ in (2.21), integrating the right hand side of the resulting expression by parts and applying Lemma 3.7 gives

$$
\left(a^{-1} \operatorname{curl} \psi, \operatorname{curl} \beta_{\gamma}\right)=\mu|\gamma|^{2} \frac{\partial}{\partial \hat{\boldsymbol{t}}_{\gamma}^{K^{\prime}}}\left(\frac{\partial u_{n c \mid K^{\prime}}}{\partial \hat{\boldsymbol{t}}_{\gamma}^{K^{\prime}}}\right)-\left(\beta_{\gamma}, \frac{\partial q}{\partial \hat{\boldsymbol{t}}_{\gamma}^{K^{\prime}}}\right)_{\gamma} .
$$

Combining this with (4.11) yields

$$
u_{n c \mid K^{\prime}}\left(\boldsymbol{x}_{n}\right)-q\left(\boldsymbol{x}_{n}\right)=\left(a^{-1} \operatorname{curl} \psi, \operatorname{curl} \beta_{\gamma}\right)+\left(\beta_{\gamma}, \frac{\partial\left(q-q_{I}\right)}{\partial \hat{\boldsymbol{t}}_{\gamma}^{K^{\prime}}}\right)_{\gamma}
$$

which we can add to (4.9) then insert the resulting expression into (4.10) to obtain

$$
u_{n c \mid K}\left(\boldsymbol{x}_{n}\right)-\mathcal{S}\left(u_{n c}\right)\left(\boldsymbol{x}_{n}\right)=\left(a^{-1} \operatorname{curl} \psi, \operatorname{curl} \beta_{K \Gamma_{D}, n}\right)+\left(\beta_{\gamma}, \frac{\partial\left(q-q_{I}\right)}{\partial \hat{\boldsymbol{t}}_{\gamma}^{K^{\prime}}}\right)_{\gamma}
$$

where $\beta_{K \Gamma_{D}, n}=\beta_{K K^{\prime}}+\beta_{\gamma}$. Observing that $\left(\frac{\partial \beta_{\gamma}}{\partial \hat{\boldsymbol{t}}_{\gamma}^{K^{\prime}}}, c\right)_{\gamma}=0$ for all $c \in \mathbb{R}$ and integrating by parts allows us to say that

$$
\left(\beta_{\gamma}, \frac{\partial\left(q-q_{I}\right)}{\partial \hat{\boldsymbol{t}}_{\gamma}^{K^{\prime}}}\right)_{\gamma}=-\left(\frac{\partial \beta_{\gamma}}{\partial \hat{\boldsymbol{t}}_{\gamma}^{K^{\prime}}}, q-q_{I}-c\right)_{\gamma}
$$

with $c \in \mathbb{R}$ chosen so that $\left\|q-q_{I}-c\right\|_{L_{2}(\gamma)} \leq C h_{K^{\prime}}^{1 / 2}$ osc $(q, \gamma)$. The result then follows by combining the above and applying Cauchy-Schwarz and the estimates $\left\|a^{-1 / 2} \operatorname{curl} \beta_{K \Gamma_{D}, n}\right\|_{L_{2}\left(\Omega_{n}\right)} \leq C a_{K \Gamma_{D}, n}^{-1 / 2}$ and $\left\|\frac{\partial \beta_{\gamma}}{\partial \hat{\boldsymbol{t}}_{\gamma}^{K^{\prime}}}\right\|_{L_{2}(\gamma)} \leq C h_{K^{\prime}}^{-1 / 2}$.

Lemma 4.3. Let $\tilde{K}$ denote the patch formed from element $K$ and those elements sharing a common vertex with element $K$ and let $\mathcal{E}_{K}$ denote the set of edges having any vertex of $K$ as an endpoint. Then there exists a positive constant $c$, depending only on the shape of the elements, such that

$$
c \Psi_{K}^{2} \leq \Upsilon_{K}\left(a^{-1} \operatorname{curl} \psi, \operatorname{curl} \psi\right)_{\tilde{K}}+a_{K} \sum_{\gamma \subset \mathcal{E}_{K} \cap \Gamma_{D}} \operatorname{osc}^{2}(q, \gamma) .
$$

Proof. By a standard argument we have that

$$
\Psi_{K}^{2} \leq C\left(\left\|u_{n c}-\mathcal{S}\left(u_{n c}\right)\right\|_{K}^{2}+a_{K} \sum_{\gamma \subset \partial K \cap \Gamma_{D}} \operatorname{osc}^{2}(q, \gamma)\right)
$$


and by the equivalence of norms in finite dimensions we have that

$$
\left\|u_{n c}-\mathcal{S}\left(u_{n c}\right)\right\|_{K}^{2} \leq C \sum_{n \in \mathcal{N}(K)} a_{K}\left|u_{n c \mid K}\left(\boldsymbol{x}_{n}\right)-\mathcal{S}\left(u_{n c}\right)\left(\boldsymbol{x}_{n}\right)\right|^{2} .
$$

The result then follows by inserting the estimates proved in Lemma 4.2 and observing that

$$
\sum_{n \in \mathcal{N}(K) \cap \mathcal{N}\left(\Gamma_{D}\right)} \frac{a_{K}}{a_{K \Gamma_{D}, n}}+\sum_{K^{\prime} \subset \tilde{K} \backslash K} \frac{a_{K} \omega_{K^{\prime}, n}^{2}}{a_{K K^{\prime}}} \leq \Upsilon_{\Gamma_{D}}+\sum_{K^{\prime} \subset \tilde{K} \backslash K} \Upsilon_{K K^{\prime}}=\Upsilon_{K} .
$$

\section{Summary AND NUmerical EXAmples}

5.1. Summary of main results. Our bounds on the broken energy norm of the error $e$ in the Fortin-Soulie finite element approximation are summarised in the following theorem.

Theorem 5.1. Let $\Phi_{K}\left(\beta_{K}\right)$ and $\Psi_{K}$ be defined as in Lemma 3.5 and Lemma 4.1 respectively. Then, the broken energy norm of the total error e can be estimated as

$$
\|e\|^{2} \leq \sum_{K \in \mathcal{P}}\left(\Phi_{K}^{2}\left(\beta_{K}\right)+\Psi_{K}^{2}\right)
$$

Also, there exists a positive constant $c$, which is independent of any mesh-size, such that

$$
\begin{aligned}
c\left(\Phi_{K}^{2}\left(\beta_{K}\right)+\Psi_{K}^{2}\right) \leq & \Upsilon_{K}\|e\|_{\tilde{K}}^{2}+a_{K} \sum_{\gamma \subset \mathcal{E}_{K} \cap \Gamma_{D}} \operatorname{osc}^{2}(q, \gamma) \\
& +a_{K}^{-1} \sum_{K^{\prime} \subset \tilde{K}} \operatorname{osc}^{2}\left(f, K^{\prime}\right)+a_{K}^{-1} \sum_{\gamma \subset \partial K \cap \Gamma_{N}} \operatorname{osc}^{2}(g, \gamma)
\end{aligned}
$$

where $\tilde{K}$ denotes the patch formed from element $K$ and those elements sharing a common vertex with element $K, \mathcal{E}_{K}$ denotes the set of edges having any vertex of $K$ as an endpoint and $\Upsilon_{K}$ is defined in (2.18).

Proof. Follows from Lemma 2.2. Lemma 3.5. Lemma 3.9. Lemma 4.1 and Lemma 4.3 by observing that $\hat{K} \subset \tilde{K}$.

The evaluation of $\Psi_{K}$ defined in Lemma 4.1 is straightforward. For the evaluation of $\Phi_{K}\left(\beta_{K}\right)$, the following result will be useful.

Lemma 5.2. Let the vertices, edges, tangent and normal vectors of element $K \in \mathcal{P}$ be enumerated and have direction as shown in Figure 2 where for $i=1,2,3,\left|\boldsymbol{t}_{i}\right|=$ $\left|\boldsymbol{n}_{i}\right|=\left|\gamma_{i}\right|$. Let

$$
\vec{V}=\left[\begin{array}{c}
\operatorname{grad}\left(P_{K} f\right) \cdot\left(\boldsymbol{x}_{1}-\boldsymbol{x}_{K}\right) \\
\operatorname{grad}\left(P_{K} f\right) \cdot\left(\boldsymbol{x}_{2}-\boldsymbol{x}_{K}\right) \\
\operatorname{grad}\left(P_{K} f\right) \cdot\left(\boldsymbol{x}_{3}-\boldsymbol{x}_{K}\right) \\
\left|\gamma_{1}\right|^{2} \alpha_{\gamma_{1}}^{K} \frac{\partial}{\partial \hat{\boldsymbol{t}}_{1}}\left[a \frac{\partial u_{n c}}{\partial \hat{n}}\right]_{\gamma_{1}} \\
\left|\gamma_{2}\right|^{2} \alpha_{\gamma_{2}}^{K} \frac{\partial}{\partial \hat{t}_{2}}\left[a \frac{\partial u_{n c}}{\partial \hat{n}}\right]_{\gamma_{2}} \\
\left|\gamma_{3}\right|^{2} \alpha_{\gamma_{3}}^{K} \frac{\partial}{\partial \hat{\boldsymbol{t}}_{3}}\left[a \frac{\partial u_{n c}}{\partial \hat{\boldsymbol{n}}}\right]_{\gamma_{3}}
\end{array}\right]
$$


where $\boldsymbol{x}_{K}$ is the centroid of $K$ and $P_{K} f,\left[a \frac{\partial u_{n c}}{\partial \tilde{\boldsymbol{n}}}\right]_{\gamma}$ and $\alpha_{\gamma}^{K}$ are defined in Section 2.5. (3.2) and (3.4) respectively. Also, let

$$
\boldsymbol{S}=\frac{1}{1440}\left[\begin{array}{cc}
-\frac{|K|}{45} \boldsymbol{A} & \boldsymbol{B} \\
\boldsymbol{B}^{T} & \frac{15}{|K|} \boldsymbol{C}
\end{array}\right]
$$

where, with $Q=\frac{1}{2} \sum_{i=1}^{3}\left|\gamma_{i}\right|^{2}, \boldsymbol{A}$ is the matrix with entries

$$
\begin{aligned}
a_{i j} & =21\left(1-6 \delta_{i j}\right) Q+\left(16+54 \delta_{i j}\right)\left(\boldsymbol{t}_{i} \cdot \boldsymbol{t}_{j}\right), \\
\boldsymbol{B} & =\left[\begin{array}{ccc}
\left|\gamma_{2}\right|^{2}-\left|\gamma_{3}\right|^{2} & -s_{12} & s_{13} \\
s_{21} & \left|\gamma_{3}\right|^{2}-\left|\gamma_{1}\right|^{2} & -s_{23} \\
-s_{31} & s_{32} & \left|\gamma_{1}\right|^{2}-\left|\gamma_{2}\right|^{2}
\end{array}\right],
\end{aligned}
$$

where $s_{i j}=2\left|\gamma_{i}\right|^{2}+5\left|\gamma_{j}\right|^{2}+6\left(\boldsymbol{t}_{i} \cdot \boldsymbol{t}_{j}\right)$, and $\boldsymbol{C}$ is the matrix with entries $c_{i j}=$ $\delta_{i j} Q+\left(1-\delta_{i j}\right)\left(\boldsymbol{t}_{i} \cdot \boldsymbol{t}_{j}\right)$. Then

$$
\left\|\boldsymbol{\sigma}_{K}\right\|_{L_{2}(K)}^{2}=\vec{V}^{T} \boldsymbol{S} \vec{V}
$$

Proof. An expression for $\left\|\boldsymbol{\sigma}_{K}\right\|_{L_{2}(K)}$ can be obtained by using the definition of $\boldsymbol{\sigma}_{K}$ and formula (2.4) to evaluate $\left(\boldsymbol{\sigma}_{K}, \boldsymbol{\sigma}_{K}\right)$. This expression can then be simplified using the identity $\boldsymbol{t}_{1}+\boldsymbol{t}_{2}+\boldsymbol{t}_{3}=\mathbf{0}$ and finally manipulated into the above form.

5.2. Numerical examples. We illustrate the theoretical results with three examples: one with a smooth solution, one where the coefficient $a$ undergoes jumps of varying sizes and one where the oscillation of $f$ is non-zero, the coefficient $a$ undergoes jumps and the gradient of the true solution displays highly singular behaviour at the origin.

The definition of $\Phi_{K}$ involves an arbitrary function $\beta_{K} \in H_{0}^{1}(K)$. This may simply be chosen to vanish, but alternative choices may be made in the hope of tightening the error bounds. In the following example we also present the results obtained by choosing $\beta_{K}$ to be cubic, quartic and quintic polynomials supported on the element. When reporting numerical results we let Ndofs denote the number of degrees of freedom, for $n=0,3,4,5$, let $\eta_{n}^{2}=\sum_{K \in \mathcal{P}}\left(\Phi_{K}^{2}\left(\beta_{K}^{(n)}\right)+\Psi_{K}^{2}\right)$ where $\beta_{K}^{(n)} \in H_{0}^{1}(K) \cap \mathbb{P}_{n}(K)$ is chosen to minimise $\Phi_{K}$ and let the effectivity index of the estimator be denoted by $\vartheta_{n}=\frac{\eta_{n}}{\|e\|}$. Note that minimising over $H_{0}^{1}(K) \cap \mathbb{P}_{0}(K)=0$ is equivalent to omitting the bubble correction.

5.3. Example 1. Our first example is the problem $-\operatorname{div}(\operatorname{grad} u)=-6 x$ in $\Omega=$ $(0,1)^{2}$, with the true solution given by $u=x^{3}$. Dirichlet conditions are applied on the vertical edges while Neumann conditions are chosen on the horizontal edges. The initial mesh is formed by subdividing the domain into two elements by joining the top left vertex to the bottom right vertex. The mesh was then uniformly refined whereby each triangle is subdivided into four subtriangles at each step.

It can be seen from Figure 3 that there is a notable difference if a cubic bubble is introduced, but further enrichments to quartic and quintic bubbles have little effect. Consequently, we shall apply a cubic bubble correction in the remaining examples. 


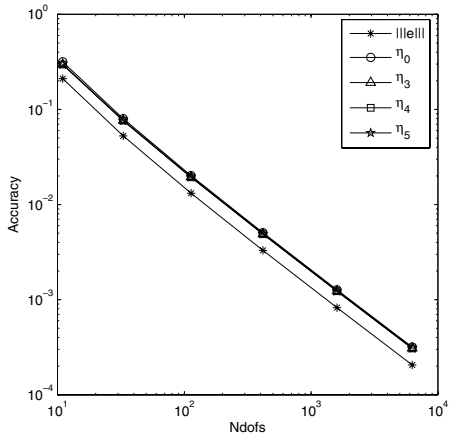

(a)

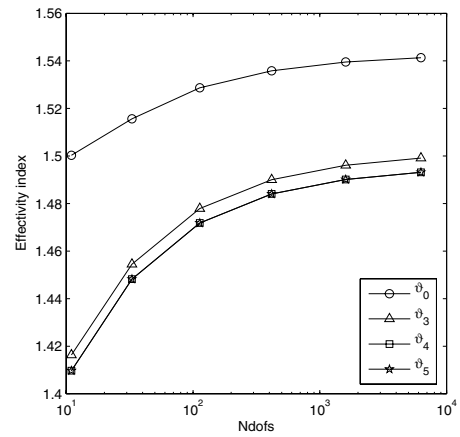

(b)

Figure 3. Performance (a) and effectivity index (b) of estimators for Example 1.

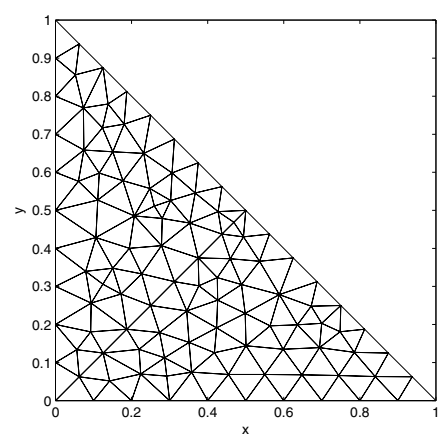

(a)

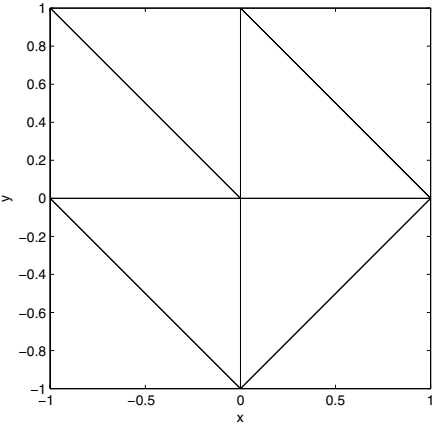

(b)

FIgURE 4. Initial mesh for Example 2(a) and Example 3(b).

5.4. Example 2. Our second example is for the problem $-\operatorname{div}(a \operatorname{grad} u)=f$ in the region $\Omega$ bounded by the lines $x=0, y=0$ and $x+y=1$ with boundary condition $u=0$ on $\Gamma_{D}=\partial \Omega$, where $f$ is chosen so that the true solution is given by $u=x y(1-x-y)$. We shall vary the value taken by $a$ in the region to the left of the line $y=x$ and keep $a=1$ in the region to the right of this line. The mesh used for this example is shown in Figure 4(a). The purpose of this example is to show that, although any choice of weights satisfying $\alpha_{\gamma}^{K}+\alpha_{\gamma}^{K^{\prime}}=1$ where $\gamma=\partial K \cap \partial K^{\prime}$ will provide an upper bound for the conforming error, the estimator with weights defined in (3.4) is better than choosing equal weights.

It can be seen from Figure 5 that if the weights on interior edges are chosen to be equal and there are large jumps in the permeability coefficient $a$, then the estimator is extremely pessimistic. In contrast the weights defined in (3.4) give an effectivity index of approximately 1.6 regardless of the magnitude of the jump in the permeability. 


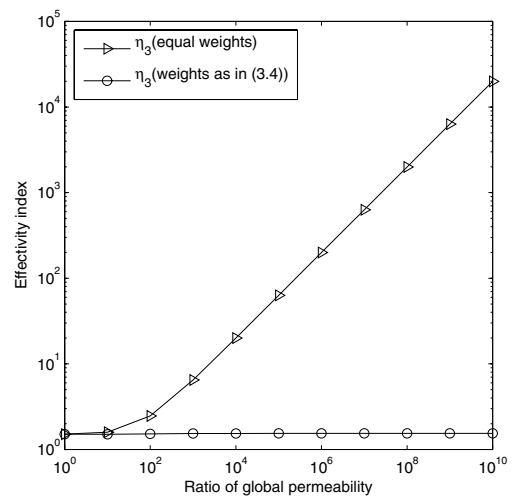

FiguRE 5. Effectivity index of estimators for Example 2 with equal weights and weights defined in (3.4).

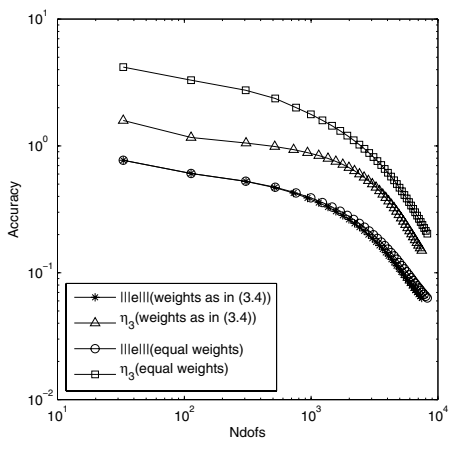

(a)

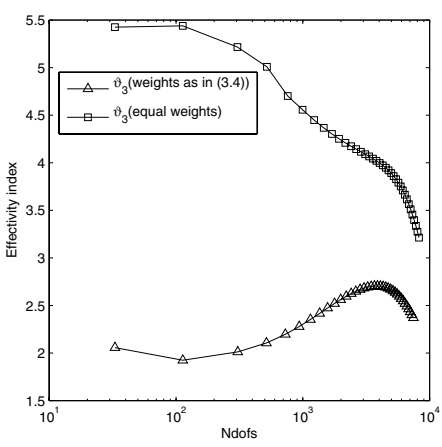

(b)

Figure 6. Performance (a) and effectivity index (b) of estimators for Example 3 with equal weights and weights defined in (3.4).

5.5. Example 3. For our final example we look at the performance of the estimator for the problem $-\operatorname{div}\left(a \operatorname{grad} u^{*}\right)=f$ in the region $\Omega=(0,1)^{2}$ with homogeneous Dirichlet data on $\Gamma_{D}=\partial \Omega$. The function $f$ has been chosen so that the exact solution to this problem is $u^{*}=\left(1-x^{2}\right)\left(1-y^{2}\right) u$ where $u$ is the solution to the Kellogg problem from the Example: Discontinuous Coefficients in 17] where $a$ is also defined to take the value $161.4 \ldots$ in the first and third quadrants and 1 in the second and fourth quadrants. The gradient of the true solution displays highly singular behaviour at the origin since $\operatorname{grad} u^{*}=O\left(r^{-9 / 10}\right)$. The initial mesh used for this example is shown in Figure 4 (b). The mesh was then adaptively refined whereby a bulk criterion [12] was used to refine the mesh on the smallest number of elements such that the sum of the contributions toward the estimator from these elements exceeded $50 \%$ of the value of the estimator. 
From Figure 6 it can be seen that while the ratio of the estimator to the broken energy norm of the true error is not as small as it was in the previous example it only overestimates the error by a factor of at most 2.71 when the weights defined in (3.4) are used. As in the previous example, the performance of the estimator is not as good when equal weights are used in the evaluation of the upper bound for the conforming error.

\section{REFERENCES}

[1] M. Ainsworth, Robust a posteriori error estimation for nonconforming finite element approximation, SIAM J. Numer. Anal. 42 (2005), no. 6, 2320-2341 (electronic). MR2139395 (2006j:65331)

[2] A posteriori error estimation for discontinuous Galerkin finite element approximation, SIAM J. Numer. Anal. 45 (2007), no. 4, 1777-1798 (electronic). MR2338409

[3] M. Ainsworth and J. T. Oden, A Posteriori Error Estimation in Finite Element Analysis, Pure and Applied Mathematics, Wiley-Interscience, John Wiley \& Sons, New York, 2000. MR2003b:65001

[4] A. Alonso, Error estimators for a mixed method, Numer. Math. 74 (1996), no. 4, 385-395. MR.1414415 (97g:65212)

[5] I. Babuska and T. Strouboulis, The Finite Element Method and its Reliability, Numerical Mathematics and Scientific Computation, Oxford University Press, Oxford, 2001. MR.1857191 (2002k:65001)

[6] C. Bernardi and R. Verfürth, Adaptive finite element methods for elliptic equations with non-smooth coefficients, Numer. Math. 85 (2000), no. 4, 579-608. MR 1771781|(2001e:65177)

[7] D. J. Blacker, A robust a posteriori error estimate for the Fortin-Soulie finite-element method, Comput. Math. Appl. 48 (2004), no. 12, 1863-1876. MR2116962 (2005m:65244)

[8] S. C. Brenner, K. Wang, and J. Zhao, Poincaré-Friedrichs inequalities for piecewise $H^{2}$ functions, Numer. Funct. Anal. Optim. 25 (2004), no. 5-6, 463-478. MR2106270 (2005i:65178)

[9] C. Carstensen, A posteriori error estimate for the mixed finite element method, Math. Comp. 66 (1997), no. 218, 465-476. MR1408371 (98a:65162)

[10] C. Carstensen, S. Bartels, and S. Jansche, A posteriori error estimates for nonconforming finite element methods, Numer. Math. 92 (2002), no. 2, 233-256. MR1922920(2003g:65139)

[11] E. Dari, R. Duran, C. Padra, and V. Vampa, A posteriori error estimators for nonconforming finite element methods, RAIRO Modél. Math. Anal. Numér. 30 (1996), no. 4, 385-400. MR.1399496 (97f:65066)

[12] W. Dörfler, A convergent adaptive algorithm for Poisson's equation, SIAM J. Numer. Anal. 33 (1996), no. 3, 1106-1124. MR1393904 (97e:65139)

[13] M. Fortin and M. Soulie, A nonconforming piecewise quadratic finite element on triangles, Internat. J. Numer. Methods Engrg. 19 (1983), no. 4, 505-520. MR702056 (84g:76004)

[14] R. H. W. Hoppe and B. Wohlmuth, Element-oriented and edge-oriented local error estimators for nonconforming finite element methods, RAIRO Modél. Math. Anal. Numér. 30 (1996), no. 2, 237-263. MR.1382112 (97e:65124)

[15] H. Lee and D. Sheen, Basis for the quadratic nonconforming triangular element of Fortin and Soulie, Int. J. Numer. Anal. Model. 2 (2005), no. 4, 409-421. MR2177630 (2006j:65355)

[16] L. D. Marini, An inexpensive method for the evaluation of the solution of the lowest order Raviart-Thomas mixed method, SIAM J. Numer. Anal. 22 (1985), no. 3, 493-496. MR787572 (86g:65214)

[17] P. Morin, R. H. Nochetto, and K. G. Siebert, Data oscillation and convergence of adaptive FEM, SIAM J. Numer. Anal. 38 (2000), no. 2, 466-488 (electronic). MRMR1770058 (2001g:65157)

[18] S. Nicaise, A posteriori error estimations of some cell-centered finite volume methods, SIAM J. Numer. Anal. 43 (2005), no. 4, 1481-1503 (electronic). MR2182137(2006j:65316)

[19] C. Padra, A posteriori error estimators for nonconforming approximation of some quasiNewtonian flows, SIAM J. Numer. Anal. 34 (1997), no. 4, 1600-1615. MR1461798 (98h:65050)

[20] L. E. Payne and H. F. Weinberger, An optimal Poincaré inequality for convex domains, Arch. Rational Mech. Anal. 5 (1960), 286-292 (1960). MR0117419 (22:8198) 
[21] M. Petzoldt, A posteriori error estimators for elliptic equations with discontinuous coefficients, Adv. Comput. Math. 16 (2002), no. 1, 47-75. MR.1888219 (2002m:65110)

[22] R. Verfürth, A Review of A Posteriori Error Estimation and Adaptive Mesh-Refinement Techniques, Wiley-Teubner, 1996.

Department of Mathematics, Strathclyde University, 26 Richmond Street, Glasgow G1 1XH, SCOTLAND

E-mail address: M.Ainsworth@strath.ac.uk

Department of Mathematics, Strathclyde University, 26 Richmond Street, Glasgow G1 1XH, SCOTLAND

E-mail address: rs.rran@maths.strath.ac.uk 